\title{
Stability Analysis of Periodic Solutions in Alternately Advanced and Retarded Neural Network Models with Impulses
}

\author{
Kuo-Shou Chiu
}

\begin{abstract}
In this paper, the global exponential stability and periodicity are investigated for impulsive neural network models with Lipschitz continuous activation functions and piecewise alternately advanced and retarded argument of generalized argument (in short IDEPCAG). The sufficient conditions for the existence and uniqueness of periodic solutions of the model are established by applying fixed point theorem and the successive approximations method. By constructing suitable differential inequalities with piecewise alternately advanced and retarded argument, some sufficient conditions for the global exponential stability of the model are obtained. Typical numerical examples with simulations are utilized to illustrate the validity and improvement in less conservatism of the theoretical results.
\end{abstract}

\section{Introduction}

Multi-variable feedback systems can exert the retroactive effect on very different time scales. Exemplifying by the extremes, according to the date of the information that is used to feedback, this action can define: (a) a continuous process or (b) one discrete process. In case (a), the growth rates of the variables are feed backed at each instant, let's say in real time. While, in case (b) there is a set of isolated dates, for example, a succession of instants in which the information is taken, in order to feedback the period between two consecutive sequence elements.

Normally and for mathematical modeling purposes, in case (a) differential equations are used and in case (b), if there is no other dynamics effect between the feedback times, difference equations can be used to express the essence of the dynamics. There are processes (real-world systems, such as some biotechnology-based ones) that can not be categorized into types (a) or (b), as they combine characteristics of both types of scales among other particular effects.

Lately, the new type of feedback systems shows a combination of characteristics from both the continuous-time and the discrete-time systems, which is neither continuous time

Received March 9, 2021; Accepted September 8, 2021.

Communicated by Cheng-Hsiung Hsu.

2020 Mathematics Subject Classification. 92B20, 34A37, 34K13, 34K20, 34K34, 26D10.

Key words and phrases. impulsive neural networks, piecewise constant argument of generalized type, periodic solutions, global exponential stability, Gronwall integral inequality.

This research was in part supported by PGI 03-2020 DIUMCE. 
nor purely discrete-time; among them are dynamical systems with impulses and systems with piecewise constant arguments. This leads to the use of hybrid type equations, for example, the impulsive differential equations with piecewise constant arguments (in abbreviation: IDEPCA) were first considered by Wiener and Lakshmikantham [38] in 2000 and differential equations with piecewise constant argument without impulsive effect (in short, DEPCA) were studied by Shah and Wiener [34] and Wiener [36] in 1984; and have been investigated by many authors. Diverse applications of the DEPCA theory are discussed in [16, 20, 24, 30, 33, 39]. We highlight the book of J. Wiener [37], pioneer of DEPCA, that recollects much of the research done in DEPCA. In the case, DEPCA of generalized type, were discussed extensively in $[1,2,11,12,14,15,17,32]$.

When scales are mixed these feedback systems can be visualized as control systems, in that, one scale represents the intrinsic of the process and the other is external intervention. However, based on internal parameters. As an example, mentioned in Busenberg and Cooke [4], is the example of the stabilization of hybrid control systems with feedback delay, in which a hybrid system is a dynamical system that presents both continuous and discrete dynamic behavior. The hybrid control systems are very interesting, which depend on the attributes and simplifications of modeling on the process, being the most usual, to represent the intrinsic process with the continuous time scale and to reflect the intervention from the external environment to the system with the discrete scale.

Note that, either as a feedback system or as a system under control, the questions of interest usually refer to the behavior of the variables in the long term, in particular looking for specific patterns according to values in the space of feasible parameters. For reasons of practical necessity for the modeled processes, the most recurrently sought behavior is stability, in some sense, for example, seen as convergence to a steady state or towards dynamic cycles.

As far as the present work is concerned, we are interested in systems of $n$-variables $x(t)$ with hybrid type feedback, i.e., in which to the properly continuous retroaction, that is, the differential system $x^{\prime}(t)=\mathcal{F}(t, x(t))$, is added another action $\mathcal{G}(\cdot, \gamma(\cdot), x(\gamma(\cdot)))$ of constant type during intervals of time $I_{\kappa}=\left[t_{\kappa}, t_{\kappa+1}\right), \kappa \in \mathbb{N}$, whose edge points are a predetermined sequence of times $\left\{t_{\kappa}\right\}$, this from internal information obtained in said sequence and $\mathfrak{J}_{\kappa}$, are impulsive effects at the moments $t_{\kappa}$. Hence

$$
\begin{aligned}
x^{\prime}(t) & =\mathcal{F}(t, x(t))+\mathcal{G}(t, \gamma(t), x(\gamma(t))), & & t \neq t_{\kappa}, \\
\Delta x\left(t_{\kappa}\right) & =\mathfrak{J}_{\kappa}\left(x\left(t_{\kappa}^{-}\right)\right), & & \kappa \in \mathbb{N},
\end{aligned}
$$

where the timer is given by $\gamma(t)=\gamma_{\kappa}, t_{\kappa} \leq \gamma_{\kappa}<t_{\kappa+1}$, if $t \in I_{\kappa}$.

In 1988, Chua et al. [18] presented a new class of information-processing systems referred to as cellular neural networks $(\mathrm{CNNs})$. It is known that the study of the stability 
of CNNs, DCNNs (delayed CNNs) and ICNNs (CNNs with impulses) is an important problem in theory and application. Many essential aspects of these networks, such as qualitative features of stability, periodicity, oscillation, and convergence problems have been examined by many other authors (see $3,5,5,7,10,19,20,25,29,31,35,40,42]$ and the references cited therein).

In 2000, J. Cao [5] proposed the problem of neural networks with transmission delays by using the Lyapunov method. Afterwards, considering theory of $M$-matrices, some stability criteria were established for delayed Hopfield neural networks [7] and the convergence behavior of a unique equilibrium of ICNNs was derived from 19 .

In 2003, in view of Halanay-type inequalities and the Lyapunov methods, Mohamad and Gopalsamy [29] discussed the stability of DCNNs with continuous and discrete time; Zhou and $\mathrm{Hu}$ [42] (2008) studied periodic and stability conditions for DCNNs with variable and distributed delays. In 2004, by using Mawhin's coincidence degree theory and Gronwall's inequality, Liu and Liao 27 investigated DCNNs with periodic coefficients.

J. H. Park [31] (2006), B. Wang et al. 35] (2008), Zhang [41 (2009), O. M. Kwona et al. 25] and T. Li [26 (2012) acquired some delay-dependent stability criteria for interval time-varying delays neural networks, by constructing a Lyapunov-Krasovskii functional and linear matrix inequalities. In [28,40, some criteria have been derived for high-order neural networks without and with time-varying delays, which were analyzed using the Lyapunov method and analytical technique by linear matrix inequality.

In 2006, Huang et al. 20] were the first in considering a cellular neural network defined by $1.1 \mathrm{a}$ with $\mathcal{F}(\cdot, x(\cdot))=0$ and where the $i$-th component of $\mathcal{G}(\cdot, \gamma(\cdot), x(\gamma(\cdot)))$ is given by

$$
x_{i}^{\prime}(t)=-a_{i}([t])+\sum_{j=1}^{m} b_{i j}([t]) g_{j}\left(x_{j}([t])\right)+d_{i}([t]),
$$

where $i=1,2, \ldots, m$ and $\gamma(t)=[t]$ is the greatest integer function. In this case, $x^{\prime}(t)$ depends during all the interval $[n, n+1), n$ an integer number, only of the value of functions defined at instant $n$.

In 2007, Huang et al. 23 investigated the following hybrid neural network with piecewise constant delay

$$
x_{i}^{\prime}(t)=-a_{i}(t)+\sum_{j=1}^{m} b_{i j}(t) g_{j}\left(x_{j}\left(\left[\frac{t}{\kappa}\right] \kappa\right)\right)+d_{i}(t),
$$

where $i=1,2, \ldots, m, \gamma(t)=\left[\frac{t}{\kappa}\right] \kappa$ and $\kappa$ is a fixed positive real number. In this case, $x^{\prime}(t)$ depends during all the interval $[n \kappa,(n+1) \kappa), n$ an integer number. So, equations type (1.1a), with $\gamma(\cdot)$ a constant delay of generalized type, are named differential equation with generalized piecewise constant delay (DEGPCD). The theory of the DEGPCD with impulsive effect (IDEGPCD) has been investigated by few authors. See [1, 2, 9, 12, 
We say that a deviation argument is of piecewise alternately advanced and retarded argument, and denote $\gamma(t)=\gamma_{\kappa}, t_{\kappa} \leq \gamma_{\kappa}<t_{\kappa+1}$, if $t \in I_{\kappa}$, for all $\kappa \in \mathbb{N}$. One can easily see that, the deviation argument $\ell(t)=t-\gamma(t)$ is assumed to be negative for $t_{\kappa}<t<\gamma_{\kappa}$ and positive for $\gamma_{\kappa}<t<t_{\kappa+1}, \kappa \in \mathbb{N}$. Therefore, $1.1 \mathrm{a}$ is of considerable interest: on each interval $\left[t_{\kappa}, t_{\kappa+1}\right)$ it is of alternately advanced and retarded type. Equation (1.1a) is of advanced type on $I_{\kappa}^{+}=\left[t_{\kappa}, \gamma_{\kappa}\right]$ and retarded type on $I_{\kappa}^{-}=\left(\gamma_{\kappa}, t_{\kappa+1}\right)$. So, equations type 1.1a), with $\gamma(\cdot)$ of alternately advanced and retarded type, are named differential equation with piecewise alternately advanced and retarded argument of generalized type (DEPCAG). The equations type can represent anticipatory models. Note that the scientific mathematical community around the DEPCAG with impulsive effect (IDEPCAG) is very limited. See 6, 13.

In the present work, we will consider a case of the IDEPCAG system $1.1 \mathrm{a})-1.1 \mathrm{~b})$ of more linear nature, but also combining information of the instant with information of the past, present, future and impulsive effect. This is, $1.1 \mathrm{a})-(1.1 \mathrm{~b})$ with

$$
\begin{aligned}
x^{\prime}(t) & =-A(t) x(t)+B(t) f(x(t))+C(t) g(x(\gamma(t)))+D(t), & & t \neq t_{\kappa}, \\
\Delta x\left(t_{\kappa}\right) & =\mathfrak{J}_{\kappa}\left(x\left(t_{\kappa}^{-}\right)\right), & & \kappa \in \mathbb{N},
\end{aligned}
$$

where $A(t)=\operatorname{diag}\left\{a_{i}(t)\right\}, B=\left\{b_{i j}(t)\right\}$ and $C=\left\{c_{i j}(t)\right\}$ are $n \times n$ real continuous matrix functions, $D(t)=\left\{d_{i}(t)\right\}$ is $n \times 1$ real continuous matrix function and $\mathfrak{J}_{k}$ is $n \times 1$ real continuous matrix function which represents the impulsive effects.

Notice that, to know information about the behavior of solutions of $(1.2 \mathrm{a}-(1.2 \mathrm{~b})$, as a mathematical problem, has an historical evolution, we can point out that

(1) In [1] (2010), stability criterion for the equilibrium and the periodic solution of the IDEGPCD system are presented with the linearization method.

(2) In [10] (2017), sufficient conditions for the periodic solution of the IDEPCA system are established with the Green's function and fixed point theorem.

(3) In [12] (2021), stability criterion for the equilibrium of the IDEGPCD system are presented with the linear approximation method.

(4) In [14 (2021), sufficient conditions for the periodic solution of the IDEGPCD system are established.

The novelty of our work is to present new and simple sufficient conditions ensuring existence, uniqueness and global exponential stability of the periodic solutions for impulsive neural network models with piecewise alternately advanced and retarded argument of generalized type (ICNN models with the IDEPCAG system). The proposed criteria 
extend the results of the previous literature. The method is given by the traditional and tailored route of a: IDEPCAG's Gronwall inequality and Banach contraction principle.

This paper is organized as follows. In Section 2, we focus on some preliminary results which will be used in the existence and stability of a unique $\omega$-periodic solution of the ICNN models with the IDEPCAG system. In Section 3.1, we establish the sufficient conditions for existence and uniqueness of the $\omega$-periodic solutions of the ICNN models with the IDEPCAG system 11.2a - 1.2b). In Section 3.2, we derive some sufficient conditions for the global exponential stability of a unique $\omega$-periodic solution of the ICNN models with the IDEPCAG system 1.2a - 1.2b). In Section 4, two examples and the numerical simulations are given to demonstrate the validity of our results. The conclusions are drawn in Section 5 ,

\section{Preliminaries}

In this section, we will focus on presenting some preliminary concepts and propositions, which will be used in the proofs about existence and stability of a unique $\omega$-periodic solution of the ICNN models with the IDEPCAG system.

The system under study is a ICNN model with piecewise alternately advanced and retarded argument of generalized type. Where, the state of the $i$-th, $1 \leq i \leq n$, neuron at time $t>0$ is given by

$$
\begin{gathered}
x_{i}^{\prime}(t)=-a_{i}(t) x_{i}(t)+\sum_{j=1}^{n} b_{i j}(t) f_{j}\left(x_{j}(t)\right)+\sum_{j=1}^{n} c_{i j}(t) g_{j}\left(x_{j}(\gamma(t))\right)+d_{i}(t), \quad t \neq t_{k}, \\
\left.\Delta x_{i}\right|_{t_{k}}=\mathfrak{J}_{i k}\left(x_{i}\left(t_{k}^{-}\right)\right), \quad i=1,2, \ldots, n, k \in \mathbb{N}
\end{gathered}
$$

with $1 \leq i \leq n$, where

- The constant argument of generalized type is determined by a strictly increasing unbounded sequence of times $\left\{t_{\kappa}\right\}$ and the function $\gamma(\cdot)$ defined by $\gamma(t)=\gamma_{\kappa}, t_{\kappa} \leq \gamma_{\kappa}<$ $t_{\kappa+1}$, if $t \in I_{\kappa}=\left[t_{\kappa}, t_{\kappa+1}\right)$.

- The positive function $a_{i}(\cdot)$ denotes the relative rate with which the $i$-th unit resets its potential to the resting state when isolated from other units and inputs. So in (2.1a), it represents an exponential decay.

- The measure of activation of continuous type (resp. piecewise constant type) of the $j$-th neuron to its incoming potentials is given at any time by the function $f_{j}\left(x_{j}(\cdot)\right)$ $\left(\right.$ resp. $\left.g_{j}\left(x_{j}(\beta(\cdot))\right)\right)$.

- The function $b_{i j}(\cdot)$ (resp. $c_{i j}(\cdot)$ ) denotes the synaptic connection weight of continuous type (resp. piecewise type) of the unit $j$ on the unit $i$. 
- For each neuron, there is an activation flow from outside the system. It is represented by the function $d_{i}(\cdot)$ for the $i$-th one.

- $\Delta x_{i}\left(t_{k}\right)$ denotes $x_{i}\left(t_{k}\right)-x_{i}\left(t_{k}^{-}\right)$, where $x_{i}\left(t_{k}^{-}\right)=\lim _{h \rightarrow 0^{-}} x_{i}\left(t_{k}+h\right)$. Moreover, the numbers $x_{i}\left(t_{k}^{-}\right)$and $x_{i}\left(t_{k}\right)$ are, respectively, the states of the $i$-th unit before and after impulse perturbation at the moment $t_{k}, k \in \mathbb{N}$, and represents the abrupt change of the state $\mathfrak{J}_{i k}\left(x_{i}\left(t_{k}^{-}\right)\right)$at the impulsive moment $t_{k}$.

For reasons of convenience, certain assumptions are formulated below, which will be convened when necessary.

\section{Lipschitz condition.}

$\left(\mathrm{L}_{1}\right)$ The activation functions $f_{j}$ and $g_{j}$ with $f_{j}(0)=0, g_{j}(0)=0,0 \leq j \leq n$, satisfy

$$
\left|f_{j}(u)-f_{j}(v)\right| \leq \mathfrak{L}_{j}^{f}|u-v|, \quad\left|g_{j}(u)-g_{j}(v)\right| \leq \mathfrak{L}_{j}^{g}|u-v|
$$

for some positive constants $\mathfrak{L}_{i}^{f}, \mathfrak{L}_{i}^{g}$ and for all $u, v \in \mathbb{R}^{+}$.

$\left(\mathrm{L}_{2}\right)$ The impulsive operator $J_{i \kappa}, 0 \leq i \leq n, \kappa \in \mathbb{N}$, satisfies

$$
\left|\mathfrak{J}_{i \kappa}(u)-\mathfrak{J}_{i \kappa}(v)\right| \leq \mathfrak{L}_{i \kappa}^{J}|u-v|
$$

for the positive constant $\mathfrak{L}_{i \kappa}^{J}$ and for all $u, v \in \mathbb{R}^{+}$.

\section{Existence condition.}

(E) For any $\tau>0$, it is satisfied $\widehat{\kappa}(\tau):=\max \left\{\kappa_{1}, \kappa_{2}\right\}<1$, where

$$
\begin{aligned}
& \kappa^{+}:=\max _{1 \leq i \leq n}\left\{\max _{i(\tau) \leq k} \int_{t_{k}}^{\gamma_{k}} \exp \left(\int_{\gamma_{k}}^{s} a_{i}(u) d u\right)\left[\sum_{j=1}^{n} \mathfrak{L}_{j}^{f}\left|b_{i j}(s)\right|+\mathfrak{L}_{j}^{g}\left|c_{i j}(s)\right|\right] d s\right\}, \\
& \kappa^{-}:=\max _{1 \leq i \leq n}\left\{\max _{1 \leq k \leq i(\tau)} \int_{\gamma_{k}}^{t_{k+1}} \exp \left(\int_{\gamma_{k}}^{s} a_{i}(u) d u\right)\left[\sum_{j=1}^{n} \mathfrak{L}_{j}^{f}\left|b_{i j}(s)\right|+\mathfrak{L}_{j}^{g}\left|c_{i j}(s)\right|\right] d s\right\},
\end{aligned}
$$

here $i(\cdot)$ is an indexer defined by $i(t)=k$ if $t \in I_{k}=\left[t_{k}, t_{k+1}\right), k \in \mathbb{N}$.

$\left(\mathrm{E}^{\prime}\right)$ For any $\tau>0$, it is satisfied $\kappa(\tau)<1$, where

$$
\kappa:=\max _{1 \leq i \leq n}\left\{\max _{1 \leq k \leq i(\tau)} \int_{t_{k}}^{t_{k+1}} \exp \left(\int_{t_{k}}^{s} a_{i}(u) d u\right)\left[\sum_{j=1}^{n} \mathfrak{L}_{j}^{f}\left|b_{i j}(s)\right|+\mathfrak{L}_{j}^{g}\left|c_{i j}(s)\right|\right] d s\right\}
$$

here $i(\cdot)$ is an indexer defined by $i(t)=k$ if $t \in I_{k}=\left[t_{k}, t_{k+1}\right), k \in \mathbb{N}$. 
First, we prove the existence and uniqueness of solutions of the IDEPCAG system (2.1a) 2.1b). A natural extension of the original definition of a solution of the IDEPCA 8, 10,38 allows us to define a solution of the IDEPCAG system.

Definition 2.1. A function $x$ is a solution of the IDEPCAG system (2.1a) $-2.1 \mathrm{~b}$ ) in $\mathbb{R}^{+}=[0, \infty)$ if

(i) $x(t)$ is continuous for $t \in \mathbb{R}^{+}$with the possible exception of the points $t=t_{k}, k \in \mathbb{N}$.

(ii) $x(t)$ is right continuous and has left-hand limits at the points $t=t_{k}, k \in \mathbb{N}$.

(iii) $x(t)$ is differentiable and satisfies (2.1a) for any $t \in \mathbb{R}^{+}$, with the possible exception of the points $t=t_{k}, k \in \mathbb{N}$, where one-sided derivatives exist.

(iv) $x\left(t_{k}\right)$ satisfies $2.1 \mathrm{~b}, k \in \mathbb{N}$.

To study the nonlinear IDEPCAG system, we will use the approach based on the construction of an equivalent integral equation, which we give in the proposition that follows

Proposition 2.2 (Integral representation). Given a pair $\left(\tau, x_{0}\right) \in \mathbb{R}^{+} \times \mathbb{R}^{n}$, a function $x=\left(x_{1}(\cdot), \ldots, x_{n}(\cdot)\right): \mathbb{R}^{+} \rightarrow \mathbb{R}^{n}$ such that $x(\tau)=x_{0}=\left(x_{1}(\tau), \ldots, x_{n}(\tau)\right)^{T}$ is a solution of the IDEPCAG system 2.1a -2.1b in the sense of Definition 2.1 if and only if their coordinates satisfy on $\mathbb{R}^{+}$the following set of integral equations

$$
\begin{aligned}
x_{i}(t)= & e^{-\int_{\tau}^{t} a_{i}(s) d s} x_{i}(\tau) \\
& +\int_{\tau}^{t} e^{-\int_{s}^{t} a_{i}(u) d u}\left[\sum_{j=1}^{n} b_{i j}(s) f_{j}\left(x_{j}(s)\right)+\sum_{j=1}^{n} c_{i j}(s) g_{j}\left(x_{j}(\gamma(s))\right)+d_{i}(s)\right] d s \\
& +\sum_{k=i(\tau)+1}^{i(t)} e^{-\int_{t_{k}}^{t} a_{i}(s) d s} \mathfrak{J}_{i k}\left(x_{i}\left(t_{k}^{-}\right)\right), \quad i \in\{1, \ldots, n\},
\end{aligned}
$$

or

$$
\begin{aligned}
x(t)= & \Phi(t, \tau) x_{0}+\int_{\tau}^{t} \Phi(t, s)[B(s) f(x(s))+C(s) g(x(\gamma(s)))+D(s)] d s \\
& +\sum_{k=i(\tau)+1}^{i(t)} \Phi\left(t, t_{k}\right) \mathfrak{J}_{k}\left(x\left(t_{k}^{-}\right)\right), \quad t \in \mathbb{R}^{+},
\end{aligned}
$$

where $i(t)>i(\tau), \Phi(t)$ is a fundamental solution of $u^{\prime}(t)=-A(t) u(t)$ and $\Phi(t, s)=$ $\Phi(t) \Phi^{-1}(s)$. 
Proof. Consider the interval $I_{n-1}=\left[t_{n-1}, t_{n}\right)$. If we integrate $2.1 \mathrm{a}$ on this interval it follows that

$$
\begin{aligned}
x_{i}(t)= & e^{-\int_{t_{n-1}}^{t} a_{i}(s) d s} x_{i}\left(t_{n-1}\right) \\
& +\int_{t_{n-1}}^{t} e^{-\int_{s}^{t} a_{i}(u) d u}\left[\sum_{j=1}^{n} b_{i j}(s) f_{j}\left(x_{j}(s)\right)+\sum_{j=1}^{n} c_{i j}(s) g_{j}\left(x_{j}\left(\gamma_{n-1}\right)\right)+d_{i}(s)\right] d s, \\
& i \in\{1, \ldots, n\},
\end{aligned}
$$

where $\gamma(t)=\gamma_{n-1}$ for all $t \in I_{n-1}=\left[t_{n-1}, t_{n}\right)$. Then, evaluating in $t=t_{n}$ we obtain

$$
\begin{aligned}
x_{i}\left(t_{n}^{-}\right)= & e^{-\int_{t_{n-1}}^{t_{n}} a_{i}(s) d s} x_{i}\left(t_{n-1}\right) \\
& +\int_{t_{n-1}}^{t_{n}} e^{-\int_{s}^{t_{n}} a_{i}(u) d u}\left[\sum_{j=1}^{n} b_{i j}(s) f_{j}\left(x_{j}(s)\right)+\sum_{j=1}^{n} c_{i j}(s) g_{j}\left(x_{j}\left(\gamma_{n-1}\right)\right)+d_{i}(s)\right] d s, \\
& i \in\{1, \ldots, n\} .
\end{aligned}
$$

Applying the impulsive condition $\left.\Delta x_{i}\right|_{t_{n}}=x_{i}\left(t_{n}\right)-x_{i}\left(t_{n}^{-}\right)=\mathfrak{J}_{i n}\left(x_{i}\left(t_{n}^{-}\right)\right)$it follows that

$$
\begin{aligned}
x_{i}\left(t_{n}\right)= & e^{-\int_{t_{n-1}}^{t_{n}} a_{i}(s) d s} x_{i}\left(t_{n-1}\right) \\
& +\int_{t_{n-1}}^{t_{n}} e^{-\int_{s}^{t_{n}} a_{i}(u) d u}\left[\sum_{j=1}^{n} b_{i j}(s) f_{j}\left(x_{j}(s)\right)+\sum_{j=1}^{n} c_{i j}(s) g_{j}\left(x_{j}\left(\gamma_{n-1}\right)\right)+d_{i}(s)\right] d s \\
& +\mathfrak{J}_{i n}\left(x\left(t_{n}^{-}\right)\right), \quad i \in\{1, \ldots, n\} .
\end{aligned}
$$

Similarly,

$$
\begin{aligned}
& x_{i}\left(t_{i(\tau)+1}\right) \\
= & e^{-\int_{\tau}^{t_{i(\tau)+1}} a_{i}(s) d s} x_{i}(\tau) \\
& +\int_{\tau}^{t_{i(\tau)+1}} e^{-\int_{s}^{t_{i(\tau)+1}} a_{i}(u) d u}\left[\sum_{j=1}^{n} b_{i j}(s) f_{j}\left(x_{j}(s)\right)+\sum_{j=1}^{n} c_{i j}(s) g_{j}\left(x_{j}\left(\gamma_{i(\tau)}\right)\right)+d_{i}(s)\right] d s \\
& +\mathfrak{J}_{i(\tau)+1}\left(x\left(t_{i(\tau)+1}^{-}\right)\right), \quad i \in\{1, \ldots, n\} .
\end{aligned}
$$

Then, solving the finite difference equation we obtain

$$
\begin{aligned}
x_{i}\left(t_{n}\right)= & e^{-\int_{\tau}^{t_{n}} a_{i}(s) d s} x_{i}(\tau) \\
& +\int_{\tau}^{t_{i(\tau)+1}} e^{-\int_{s}^{t_{n}} a_{i}(u) d u}\left[\sum_{j=1}^{n} b_{i j}(s) f_{j}\left(x_{j}(s)\right)+\sum_{j=1}^{n} c_{i j}(s) g_{j}\left(x_{j}\left(\gamma_{i(\tau)}\right)\right)+d_{i}(s)\right] d s
\end{aligned}
$$




$$
\begin{aligned}
& +\sum_{k=i(\tau)+1}^{n-1} \int_{t_{k}}^{t_{k+1}} e^{-\int_{s}^{t_{n}} a_{i}(u) d u} \\
& \times\left[\sum_{j=1}^{n} b_{i j}(s) f_{j}\left(x_{j}(s)\right)+\sum_{j=1}^{n} c_{i j}(s) g_{j}\left(x_{j}\left(\gamma_{k}\right)\right)+d_{i}(s)\right] d s \\
& +\sum_{k=i(\tau)+1}^{n-1} e^{-\int_{t_{k}}^{t_{n}} a_{i}(u) d u} \mathfrak{J}_{i k}\left(x\left(t_{k}^{-}\right)\right)+\mathfrak{J}_{i n}\left(x\left(t_{n}^{-}\right)\right) \\
& =e^{-\int_{\tau}^{t_{n}} a_{i}(s) d s} x_{i}(\tau)+\int_{\tau}^{t_{n}} e^{-\int_{s}^{t_{n}} a_{i}(u) d u}\left[\sum_{j=1}^{n} b_{i j}(s) f_{j}\left(x_{j}(s)\right)+d_{i}(s)\right] d s \\
& +\int_{\tau}^{t_{i(\tau)+1}}\left[e^{-\int_{s}^{t_{n}} a_{i}(u) d u} \sum_{j=1}^{n} c_{i j}(s) g_{j}\left(x_{j}\left(\gamma_{i(\tau)}\right)\right)\right] d s \\
& +\sum_{k=i(\tau)+1}^{n-1} \int_{t_{k}}^{t_{k+1}}\left[e^{-\int_{s}^{t_{n}} a_{i}(u) d u} \sum_{j=1}^{n} c_{i j}(s) g_{j}\left(x_{j}\left(\gamma_{k}\right)\right)\right] d s \\
& +\sum_{k=i(\tau)+1}^{n-1} e^{-\int_{t_{k}}^{t_{n}} a_{i}(u) d u} \mathfrak{J}_{i k}\left(x\left(t_{k}^{-}\right)\right)+\mathfrak{J}_{i n}\left(x\left(t_{n}^{-}\right)\right), \quad i \in\{1, \ldots, n\} .
\end{aligned}
$$

Next, applying last expression in 2.3 with $t \in I_{i(t)}$ we obtain

$$
\begin{aligned}
x_{i}(t)= & e^{-\int_{\tau}^{t} a_{i}(s) d s} x_{i}(\tau)+\int_{\tau}^{t} e^{-\int_{s}^{t} a_{i}(u) d u}\left[\sum_{j=1}^{n} b_{i j}(s) f_{j}\left(x_{j}(s)\right)+d_{i}(s)\right] d s \\
& +\int_{\tau}^{t_{i(\tau)+1}}\left[e^{-\int_{s}^{t} a_{i}(u) d u} \sum_{j=1}^{n} c_{i j}(s) g_{j}\left(x_{j}\left(\gamma_{i(\tau)}\right)\right)\right] d s \\
& +\sum_{k=i(\tau)+1}^{i(t)-1} \int_{t_{k}}^{t_{k+1}} e^{-\int_{s}^{t} a_{i}(u) d u}\left[\sum_{j=1}^{n} c_{i j}(s) g_{j}\left(x_{j}\left(\gamma_{k}\right)\right)\right] d s \\
& +\int_{t_{i(t)}}^{t}\left[e^{-\int_{s}^{t} a_{i}(u) d u} \sum_{j=1}^{n} c_{i j}(s) g_{j}\left(x_{j}\left(\gamma_{i(t)}\right)\right)\right] d s \\
& +\sum_{k=i(\tau)+1}^{i(t)} e^{-\int_{t_{k}}^{t} a_{i}(u) d u} \mathfrak{J}_{i k}\left(x\left(t_{k}^{-}\right)\right), \quad i \in\{1, \ldots, n\} .
\end{aligned}
$$

Finally, defining

$$
\int_{\tau}^{t} \sum_{j=1}^{n} c_{i j}(s) g_{j}\left(x_{j}(\gamma(s))\right) d s
$$




$$
\begin{aligned}
= & \int_{\tau}^{t_{i(\tau)+1}} \sum_{j=1}^{n} c_{i j}(s) g_{j}\left(x_{j}\left(\gamma_{i(\tau)}\right)\right) d s+\sum_{k=i(\tau)+1}^{i(t)-1} \int_{t_{k}}^{t_{k+1}} \sum_{j=1}^{n} c_{i j}(s) g_{j}\left(x_{j}\left(\gamma_{k}\right)\right) d s \\
& +\int_{t_{i(t)}}^{t} \sum_{j=1}^{n} c_{i j}(s) g_{j}\left(x_{j}\left(\gamma_{i(t)}\right)\right) d s
\end{aligned}
$$

and replacing it in the last expression we obtain $(2.2)$, so the proof is complete.

The following lemma, which is one of the most important tool will be used in the proofs of our results.

Lemma 2.3 (IDEPCAG's Gronwall inequality). Let $v, \alpha_{j}: \mathbb{R}^{+} \rightarrow \mathbb{R}^{+}, j=1,2$, be three functions such that $v$ is continuous with possible points of discontinuity of the first kind at $t=t_{\kappa}, \kappa \in \mathbb{N}$ and $\alpha_{j}$ are locally integrable, for which the inequality satisfying

$$
v(t) \leq \begin{cases}v(\tau)+\int_{\tau}^{t}\left[\alpha_{1}(s) v(s)+\alpha_{2}(s) v(\gamma(s))\right] d s+\sum_{\kappa=i(\tau)+1}^{i(t)} \varrho_{k} v\left(t_{\kappa}^{-}\right), & i(t)>i(\tau) \\ v(\tau)+\left|\int_{\tau}^{t}\left[\alpha_{1} v(s)+\alpha_{2} v(\gamma(s))\right] d s\right|, & i(t)=i(\tau) \\ v(\tau)+\int_{t}^{\tau}\left[\alpha_{1} v(s)+\alpha_{2} v(\gamma(s))\right] d s+\sum_{\kappa=i(t)+1}^{i(\tau)} \varrho_{k} v\left(t_{\kappa}^{-}\right), & i(t)<i(\tau)\end{cases}
$$

where $\varrho_{k}$ are non-negative constants. Then

(1) For $i(t)>i(\tau)$,

$$
v(t) \leq v(\tau)\left\{\prod_{\kappa=i(\tau)+1}^{i(t)}\left(1+\varrho_{\kappa}\right)\right\} \exp \left(\int_{\tau}^{t}\left[\alpha_{1}(s)+\frac{\alpha_{2}(s)}{1-\eta^{+}}\right] d s\right) .
$$

(2) For $i(t)=i(\tau)$,

$$
v(t) \leq v(\tau) \exp \left(\int_{\tau}^{t}\left[\alpha_{1}(s)+\frac{\alpha_{2}(s)}{1-\eta^{+}}\right] d s\right)
$$

(3) For $i(t)<i(\tau)$,

$$
v(t) \leq v(\tau)\left\{\prod_{\kappa=i(t)+1}^{i(\tau)} \frac{1}{1-\varrho_{k}}\right\} \exp \left(\int_{t}^{\tau}\left[\alpha_{1}(s)+\frac{\alpha_{2}(s)}{1-\eta^{-}}\right] d s\right),
$$

where

$$
\begin{aligned}
& \eta^{+}:=\sup _{i(\tau) \leq \kappa} \int_{t_{k}}^{\gamma_{\kappa}}\left[\alpha_{1}(s)+\alpha_{2}(s)\right] d s \leq \eta<1, \\
& \eta^{-}:=\sup _{1 \leq \kappa \leq i(\tau)} \int_{\gamma_{\kappa}}^{t_{\kappa+1}}\left[\alpha_{1}(s)+\alpha_{2}(s)\right] d s \leq \eta<1
\end{aligned}
$$

and $\max _{1 \leq \kappa \leq i(\tau)} \varrho_{k}<1$. 
Proof. First, consider $\tau \leq t$. Suppose that $\psi(t)$ is the right side of the inequality (2.4). Then $\psi(\tau)=v(\tau), v \leq \psi, \psi$ is a non-decreasing function and piecewise differentiable, and from (2.4), we have

$$
\begin{cases}\psi^{\prime}(t) \leq \alpha_{1}(t) \psi(t)+\alpha_{2}(t) \psi(\gamma(t)), & t \neq t_{\kappa}, \\ \psi\left(t_{\kappa}\right) \leq\left(1+\varrho_{\kappa}\right) \cdot \psi\left(t_{\kappa}^{-}\right), & \kappa \in \mathbb{N} .\end{cases}
$$

If $\tau \leq \ell \leq t$ with $t, \ell \in I_{i}$, we obtain

$$
\psi(t)-\psi(r) \leq \int_{\ell}^{t}\left(\alpha_{1}(s) \psi(s)+\alpha_{2}(s) \psi\left(\gamma_{i}\right)\right) d s .
$$

With $t=\gamma_{i}, \ell=t_{i}$ in 2.9 for $t \in I_{i}$, as $\psi$ is a non-decreasing function, we get

$$
\begin{aligned}
\psi\left(\gamma_{i}\right) & \leq \psi\left(t_{i}\right)+\int_{t_{i}}^{\gamma_{i}}\left(\alpha_{1}(s) \psi(s)+\alpha_{2}(s) \psi\left(\gamma_{i}\right)\right) d s \\
& \leq \psi\left(t_{i}\right)+\left(\int_{t_{i}}^{\gamma_{i}}\left(\alpha_{1}(s)+\alpha_{2}(s)\right) d s\right) \psi\left(\gamma_{i}\right) .
\end{aligned}
$$

By (2.7), we have

$$
\psi\left(\gamma_{i}\right) \leq \frac{\psi\left(t_{i}\right)}{1-\eta^{+}} .
$$

Take now in (2.9) with $t \in I_{i}$ and $\ell=t_{i}$, we give

$$
\begin{aligned}
\psi(t) & \leq \psi\left(t_{i}\right)+\int_{t_{i}}^{t}\left(\alpha_{1}(s) \psi(s)+\alpha_{2}(s) \psi\left(\gamma_{i}\right)\right) d s \\
& \leq \psi\left(t_{i}\right)+\int_{t_{i}}^{t}\left(\alpha_{1}(s) \psi(s)+\frac{\alpha_{2}(s)}{1-\eta^{+}} \psi\left(t_{i}\right)\right) d s \\
& \leq \psi\left(t_{i}\right)+\int_{t_{i}}^{t}\left\{\left(\alpha_{1}(s)+\frac{\alpha_{2}(s)}{1-\eta^{+}}\right) \psi(s)\right\} d s .
\end{aligned}
$$

Then, applying the Gronwall's lemma, we have

$$
\psi(t) \leq \psi\left(t_{i}\right) \exp \left(\int_{t_{i}}^{t}\left(\alpha_{1}(s)+\frac{\alpha_{2}(s)}{1-\eta^{+}}\right) d s\right) \quad \text { for } t \in I_{i} .
$$

By the impulsive condition 2.8, we obtain

$$
\psi\left(t_{i+1}\right) \leq\left(1+\varrho_{i+1}\right) \psi\left(t_{i}\right) \exp \left(\int_{t_{i}}^{t_{i+1}}\left(\alpha_{1}(s)+\frac{\alpha_{2}(s)}{1-\eta^{+}}\right) d s\right) .
$$

From 2.10), recursively we have

$$
v(t) \leq \psi(t) \leq \psi(\tau)\left\{\prod_{k=i(\tau)+1}^{i(t)}\left(1+\varrho_{k}\right)\right\} \exp \left(\int_{\tau}^{t}\left(\alpha_{1}(s)+\frac{\alpha_{2}(s)}{1-\eta^{+}}\right) d s\right)
$$


by $\psi(\tau)=v(\tau)$, we obtain 2.5 .

Now, if $0 \leq t \leq \tau$. Suppose that $w(t)$ is the right side of the inequality (2.4). So $w(\tau)=v(\tau), v \leq w, w$ is a non-increasing function and piecewise differentiable and from (2.4), we give

$$
w^{\prime}(t) \leq-\left[\alpha_{1}(t) w(t)+\alpha_{2}(t) w(\gamma(t))\right] \quad \text { and } \quad w\left(t_{\kappa}^{-}\right) \leq\left(1-\varrho_{\kappa}\right)^{-1} \cdot w\left(t_{\kappa}\right)
$$

If $\tau \geq \ell \geq t \geq 0$ with $t, \ell \in I_{j}$, we obtain

$$
w(t)-w(\ell) \leq-\int_{\ell}^{t}\left(\alpha_{1}(t) w(s)+\alpha_{2}(s) w\left(\gamma_{j}\right)\right) d s
$$

With $t=\gamma_{j}$, in 2.12 for $t \in I_{j}$ and $\ell=t_{j+1}^{-}$, since $w$ is a non-increasing function, we have

$$
\begin{aligned}
w\left(\gamma_{j}\right) & \leq w\left(t_{j+1}^{-}\right)-\int_{t_{j+1}}^{\gamma_{j}}\left(\alpha_{1}(s) w(s)+\alpha_{2}(s) w\left(\gamma_{j}\right)\right) d s \\
& \leq w\left(t_{j+1}^{-}\right)+\left(\int_{\gamma_{j}}^{t_{j+1}}\left(\alpha_{1}(s)+\alpha_{2}(s)\right) d s\right) w\left(\gamma_{j}\right) .
\end{aligned}
$$

By (2.7), we have

$$
w\left(\gamma_{j}\right) \leq \frac{w\left(t_{j+1}^{-}\right)}{1-\eta^{-}}
$$

Take now 2.13 in 2.12 with $t \in I_{j}$ and $\ell=t_{j+1}^{-}$, to get

$$
\begin{aligned}
w(t) & \leq w\left(t_{j+1}^{-}\right)+\int_{t}^{t_{j+1}}\left(\alpha_{1}(s) w(s)+\alpha_{2}(s) w\left(\gamma_{j}\right)\right) d s \\
& \leq w\left(t_{j+1}^{-}\right)+\int_{t}^{t_{j+1}}\left(\alpha_{1}(s) w(s)+\frac{\alpha_{2}(s)}{1-\eta^{-}} w\left(t_{j+1}^{-}\right)\right) d s \\
& \leq w\left(t_{j+1}^{-}\right)+\int_{t}^{t_{j+1}}\left(\alpha_{1}(s)+\frac{\alpha_{2}(s)}{1-\eta^{-}}\right) w(s) d s
\end{aligned}
$$

because $w$ is a non-increasing function. Then, applying the Gronwall's lemma, we have

$$
w(t) \leq w\left(t_{j+1}^{-}\right) \exp \left(\int_{t}^{t_{j+1}}\left(\alpha_{1}(s)+\frac{\alpha_{2}(s)}{1-\eta^{-}}\right) d s\right) \quad \text { for } t \in I_{j} .
$$

By 2.11) and $t=t_{j}$ we have

$$
w\left(t_{j}\right) \leq\left(1-\varrho_{j+1}\right)^{-1} w\left(t_{j+1}\right) \exp \left(\int_{t_{j}}^{t_{j+1}}\left(\alpha_{1}(s)+\frac{\alpha_{2}(s)}{1-\eta^{-}}\right) d s\right) .
$$


From 2.14, recursively we obtain

$$
\begin{aligned}
v(t) \leq w(t) & \leq\left(1-\varrho_{j+1}\right)^{-1} w\left(t_{j+1}\right) \exp \left(\int_{t}^{t_{j+1}}\left(\alpha_{1}(s)+\frac{\alpha_{2}(s)}{1-\eta^{-}}\right) d s\right) \\
& \leq\left(1-\varrho_{j+1}\right)^{-1}\left(1-\varrho_{j+2}\right)^{-1} w\left(t_{j+2}\right) \exp \left(\int_{t}^{t_{j+2}}\left(\alpha_{1}(s)+\frac{\alpha_{2}(s)}{1-\eta^{-}}\right) d s\right) \\
& \leq \cdots \\
& \leq w(\tau)\left\{\prod_{\kappa=j+1}^{i(\tau)}\left(1-\varrho_{\kappa}\right)^{-1}\right\} \exp \left(\int_{t}^{\tau}\left(\alpha_{1}(s)+\frac{\alpha_{2}(s)}{1-\eta^{-}}\right) d s\right)
\end{aligned}
$$

by $w(\tau)=v(\tau)$ we obtain (2.6). The proof is complete. The IDEPCAG's Gronwall inequality appears to be new.

Remark 2.4. If $\gamma(t)=2\left[\frac{t+1}{2}\right]$, then the inequality (2.4) is an IDEPCA's Gronwall inequality which has been studied in [10]. If $\gamma(t)=m\left[\frac{t+l}{m}\right]$ with $l<m$, then the inequality (2.4) with constant coefficients is an IDEPCA's Gronwall inequality which has been studied in 8 . If $\varrho_{\kappa} \equiv 0, \kappa \in \mathbb{N}$, then we get the inequality (2.4) without impulsive effect in [17]. So our results also extend the conclusion in them.

We can see that the ICNN models with the IDEPCAG system (2.1a) - 2.1b) do not have impulsive condition within the intervals $\left[t_{i}, t_{i+1}\right), i \in \mathbb{N}$, which is just like the DEPCAG system. Then applying the identical technique of Gronwall inequality with piecewise constant argument (see [17, 32]). We have the following proposition.

Proposition 2.5. Let the conditions $\left(\mathrm{L}_{1}\right)$ and $(\mathrm{E})$ be fulfilled. Then, given an initial condition $(\tau, \zeta) \in \mathbb{R}^{+} \times \mathbb{R}^{n}$, the ICNN model with the IDEPCAG system 2.1a 2.1b) on $\left[t_{i(\tau)}, t_{i(\tau)+1}\right)$ has a unique solution $x(\cdot)=x(\cdot, \tau, \zeta)=\left(x_{1}(\cdot), \ldots, x_{n}(\cdot)\right)^{T}$ such that $x(\tau)=\left(x_{1}^{0}, \ldots, x_{n}^{0}\right)^{T}=\zeta$.

The previous proposition assures the existence and uniqueness of solutions in a local sense. The following theorem provides the existence of a unique solution when the initial moment is an arbitrary positive real number $\tau$.

Theorem 2.6. Let the conditions $\left(\mathrm{L}_{1}\right),\left(\mathrm{L}_{2}\right)$ and $(\mathrm{E})$ be fulfilled. Then, given an initial condition $(\tau, \zeta) \in \mathbb{R}^{+} \times \mathbb{R}^{n}$, the ICNN model with the IDEPCAG system 2.1a) 2.1b) has a unique solution $x(\cdot)=x(\cdot, \tau, \zeta)=\left(x_{1}(\cdot), \ldots, x_{n}(\cdot)\right)^{T}$ such that $x(\tau)=\left(x_{1}^{0}, \ldots, x_{n}^{0}\right)^{T}=\zeta$.

Proof. Let $\tau \in \mathbb{R}^{+}$. Then we can see that $\tau \in\left[t_{i(\tau)}, t_{i(\tau)+1}\right)$. Using Proposition 2.5. the ICNN model with the IDEPCAG system 2.1a $-2.1 \mathrm{~b}$ has a unique solution $x(\cdot)=$ $x(\cdot, \tau, \zeta)=\left(x_{1}(\cdot), \ldots, x_{n}(\cdot)\right)^{T}$ on $\left[t_{i(\tau)}, t_{i(\tau)+1}\right)$ such that $x(\tau)=\left(x_{1}^{0}, \ldots, x_{n}^{0}\right)^{T}=\zeta$.

Applying the condition $2.1 \mathrm{~b})$, we have

$$
x\left(t_{i(\tau)+1}, \tau, \zeta\right)=x\left(t_{i(\tau)+1}^{-}, \tau, \zeta\right)+\mathfrak{J}_{i(\tau)+1}\left(x\left(t_{i(\tau)+1}^{-}, \tau, \zeta\right)\right) .
$$


Now, in the following interval $\left[t_{i(\tau)+1}, t_{i(\tau)+2}\right)$ the solution of the ICNN model with the IDEPCAG system 2.1a) $2.1 \mathrm{~b}$ satisfies

$$
y^{\prime}(t)=-A(t) y(t)+B(t) f(y(t))+C(t) g(y(\gamma(t)))+D(t)
$$

and the ICNN model with the IDEPCAG system 2.1a $-2.1 \mathrm{~b}$ admits a unique solution $y\left(t, t_{i(\tau)+1}, y^{0}\right)$ with the initial condition $y^{0}=x\left(t_{i(\tau)+1}, \tau, \zeta\right)$. By definition of the solution of the ICNN model $x(t, \tau, \zeta)=y\left(t, t_{i(\tau)+1}, y^{0}\right)$ on $\left[t_{i(\tau)+1}, t_{i(\tau)+2}\right)$. As $\mathbb{R}^{+}=\bigcup_{i=1}^{\infty}\left[t_{i}, t_{i+1}\right)$, this completes the proof by the mathematical induction.

From Theorem 2.6, we can derive the following particular results.

Corollary 2.7. Let us assume the conditions $\left(\mathrm{L}_{1}\right),\left(\mathrm{L}_{2}\right)$ and the following inequalities

$$
\begin{aligned}
& \kappa^{+}=\max _{1 \leq i \leq n}\left\{\sup _{i(\tau) \leq \kappa}\left(\frac{1-e^{-\underline{a}_{i} \cdot \vartheta_{\kappa}^{+}}}{\underline{a}_{i}}\right)\left[\sum_{j=1}^{n} \mathfrak{L}_{j}^{f}\left|\bar{b}_{i j}\right|+\sum_{j=1}^{n} \mathfrak{L}_{j}^{g}\left|\bar{c}_{i j}\right|\right]<1\right. \\
& \kappa^{-}=\max _{1 \leq i \leq n}\left\{\sup _{1 \leq \kappa \leq i(\tau)}\left(\frac{e^{\bar{a}_{i} \cdot \vartheta_{\kappa}^{-}}-1}{\bar{a}_{i}}\right)\left[\sum_{j=1}^{n} \mathfrak{L}_{j}^{f}\left|\bar{b}_{i j}\right|+\sum_{j=1}^{n} \mathfrak{L}_{j}^{g}\left|\bar{c}_{i j}\right|\right]\right\} 1
\end{aligned}
$$

hold, where $\bar{a}_{i}=\sup _{t \in \mathbb{R}^{+}} a_{i}(t), \underline{a}_{i}=\inf _{t \in \mathbb{R}^{+}} a_{i}(t), \bar{b}_{i j}=\sup _{t \in \mathbb{R}^{+}}\left|b_{i j}(t)\right|, \bar{c}_{i j}=\sup _{t \in \mathbb{R}^{+}}\left|c_{i j}(t)\right|$ and $\vartheta_{\kappa}^{+}=\gamma_{\kappa}-t_{\kappa}, \vartheta_{\kappa}^{-}=t_{\kappa+1}-\gamma_{\kappa}, \kappa \in \mathbb{N}$. Then, given $\left(\tau, x_{0}\right) \in \mathbb{R}^{+} \times \mathbb{R}^{n}$, there exists a unique solution $x(\cdot)=x\left(\cdot, \tau, x_{0}\right)=\left(x_{1}(\cdot), \ldots, x_{n}(\cdot)\right)^{T}$ of the IDEPCAG system 2.1a (2.1b) in the sense of Definition 2.1 such that $x(\tau)=x_{0}=\left(x_{1}^{0}, \ldots, x_{n}^{0}\right)^{T}$.

Corollary 2.8. Let us assume the conditions $\left(\mathrm{L}_{1}\right)$ and $\left(\mathrm{L}_{2}\right)$ that $a_{i}(t) \equiv a_{i}, b_{i j}(t) \equiv b_{i j}$, $c_{i j}(t) \equiv c_{i j}, d_{i}(t) \equiv d_{i}$ and the following inequalities

$$
\begin{aligned}
& \kappa^{+}=\max _{1 \leq i \leq n}\left\{\sup _{i(\tau) \leq \kappa}\left(\frac{1-e^{-a_{i} \cdot \vartheta_{\kappa}^{+}}}{a_{i}}\right)\left[\sum_{j=1}^{n} \mathfrak{L}_{j}^{f}\left|b_{i j}\right|+\sum_{j=1}^{n} \mathfrak{L}_{j}^{g}\left|c_{i j}\right|\right]<1,\right. \\
& \kappa^{-}=\max _{1 \leq i \leq n}\left\{\sup _{1 \leq \kappa \leq i(\tau)}\left(\frac{e^{a_{i} \cdot \vartheta_{\kappa}^{-}}-1}{a_{i}}\right)\left[\sum_{j=1}^{n} \mathfrak{L}_{j}^{f}\left|b_{i j}\right|+\sum_{j=1}^{n} \mathfrak{L}_{j}^{g}\left|c_{i j}\right|\right]<1\right.
\end{aligned}
$$

hold. Then, given $\left(\tau, x_{0}\right) \in \mathbb{R}^{+} \times \mathbb{R}^{n}$, there exists a unique solution $x(\cdot)=x\left(\cdot, \tau, x_{0}\right)=$ $\left(x_{1}(\cdot), \ldots, x_{n}(\cdot)\right)^{T}$ of the IDEPCAG system (2.1a)-(2.1b), but with the respective constant coefficients, in the sense of Definition 2.1 such that $x(\tau)=x_{0}=\left(x_{1}^{0}, \ldots, x_{n}^{0}\right)^{T}$.

Remark 2.9. If we consider the deviation argument that is of the constant delay of generalized type, i.e., $\gamma(t)=\gamma_{k}=t_{k}$, if $t \in\left[t_{k}, t_{k+1}\right), k \in \mathbb{N}$. The ICNN models with the 
IDEPCAG system 2.1a) - 2.1b can be reduced to the following IDEGPCD system

$$
\begin{aligned}
\frac{d x_{i}(t)}{d t}= & -a_{i}(t) x_{i}(t)+\sum_{j=1}^{n} b_{i j}(t) f_{j}\left(x_{j}(t)\right) \\
& +\sum_{j=1}^{n} c_{i j}(t) g_{j}\left(x_{j}(\beta(t))\right)+d_{i}(t), \quad t \neq t_{\kappa}, \\
\Delta x_{i}\left(t_{\kappa}\right)= & \mathfrak{J}_{i \kappa}\left(x_{i}\left(t_{\kappa}^{-}\right)\right), \quad \kappa \in \mathbb{N}
\end{aligned}
$$

with $1 \leq i \leq n$, where $\beta(t)=t_{\kappa}$ if $t \in I_{\kappa}=\left[t_{\kappa}, t_{\kappa+1}\right)$. Then we have the following observations.

(i) The ICNN models with the IDEGPCD system (the respective constant coefficients) is neither more nor less than system (1.1) in [1. Since those works not have a global IDEGPCD's Gronwall-type inequality, the results for this system have more stronger conditions, see [12, Example 1 and Remark 4.1].

(ii) The IDEPCAG's Gronwall inequality of this paper reduces to the result of the IDEG$P C D$ 's Gronwall inequality in [12, Lemma 2.1].

(iii) The condition (E) with $\kappa^{-}<1$ and $\gamma_{k}=t_{k}, k \in \mathbb{N}$, reduces to the condition ( $\mathrm{E}^{\prime}$ ) which is the same condition (E) in [14].

From Theorem 2.6 and Remark 2.9, we can conclude the following results.

Corollary 2.10. Let the conditions $\left(\mathrm{L}_{1}\right),\left(\mathrm{L}_{2}\right)$ and $\left(\mathrm{E}^{\prime}\right)$ be fulfilled. Then, given an initial condition $(\tau, \zeta) \in \mathbb{R}^{+} \times \mathbb{R}^{n}$, the ICNN models with the IDEGPCD system (2.17a)(2.17b) has a unique solution $x(\cdot)=x(\cdot, \tau, \zeta)=\left(x_{1}(\cdot), \ldots, x_{n}(\cdot)\right)^{T}$ such that $x(\tau)=$ $\left(x_{1}^{0}, \ldots, x_{n}^{0}\right)^{T}=\zeta$.

When the impulsive jumps of ICNN models with the IDEPCAG system (2.1b) are absent, ICNN models with IDEPCAG system (2.1a) reduces to the following non-impulsive system

$$
x_{i}^{\prime}(t)=-a_{i}(t) x_{i}(t)+\sum_{j=1}^{n} b_{i j}(t) f_{j}\left(x_{j}(t)\right)+\sum_{j=1}^{n} c_{i j}(t) g_{j}\left(x_{j}(\gamma(t))\right)+d_{i}(t), \quad t \in \mathbb{R}
$$

with $1 \leq i \leq n$.

Applying our results to CNN models with the DEPCAG system (2.18) and with the DEGPCD system 2.17a) without impulsive effects, we have

Corollary 2.11. Let the conditions $\left(\mathrm{L}_{1}\right)$ and $(\mathrm{E})$ be fulfilled. Then, given an initial condition $(\tau, \zeta) \in \mathbb{R}^{+} \times \mathbb{R}^{n}$, there exists a unique solution $x(\cdot)=x(\cdot, \tau, \zeta)=\left(x_{1}(\cdot), \ldots, x_{n}(\cdot)\right)^{T}$ of the CNN models with the DEPCAG system (2.18), such that $x(\tau)=\left(x_{1}^{0}, \ldots, x_{n}^{0}\right)^{T}=\zeta$. 
Corollary 2.12. Let the conditions $\left(\mathrm{L}_{1}\right)$ and $\left(\mathrm{E}^{\prime}\right)$ be fulfilled. Then, given an initial condition $(\tau, \zeta) \in \mathbb{R}^{+} \times \mathbb{R}^{n}$, there exists a unique solution $x(\cdot)=x(\cdot, \tau, \zeta)=\left(x_{1}(\cdot), \ldots, x_{n}(\cdot)\right)^{T}$ of the CNN models with the DEGPCD system (2.17a), such that $x(\tau)=\left(x_{1}^{0}, \ldots, x_{n}^{0}\right)^{T}=\zeta$.

Remark 2.13. Theorem 2.6 reduces to the existence result of [10, Theorem 3.1] with the classic piecewise alternately advanced and retarded argument $\gamma(t)=2\left[\frac{t+1}{2}\right]$. Corollary 2.8 reduces to existence results of 8 , Theorem 5] with $\gamma(t)=m\left[\frac{t+l}{m}\right]$, where $0<l<m$. Corollary 2.10 reduces to the results of [14, Theorem 3.1] with generalized piecewise constant delay.

With regard to non-impulsive effects, Corollary 2.11 reduces to the results of 17 , Theorem 1] with generalized piecewise alternately advanced and retarded argument. Corollary 2.12 reduces to the results of [15, Theorem 3.1] with generalized piecewise constant delay. Moreover Corollary 2.12 generalizes corresponding result obtained by [1, Theorem 2.1] and [2, Theorems 7.1.2 and 8.2.2] under complicated and stronger conditions. See [12, Example 1].

3. Existence and stability of periodic solutions

In this section, we will give the sufficient conditions for existence and global exponential stability of the $\omega$-periodic solution of the ICNN models with the IDEPCAG system (2.1a)2.1b. Here we assume the periodicity condition.

\section{Periodicity condition.}

(P) There exists $\omega>0$ such that

(1) $a_{i}(\cdot)>0, b_{i j}(\cdot), c_{i j}(\cdot)$ and $d_{i}(\cdot)$ are continuously periodic functions in $\mathbb{R}^{+}$with a common period $\omega$.

(2) There exists $p \in \mathbb{N}$, for which the sequences $\left\{t_{k}\right\}_{k \in \mathbb{N}},\left\{\gamma_{k}\right\}_{k \in \mathbb{N}}$ and $\left\{J_{k}\right\}_{k \in \mathbb{N}}$, satisfies the $(\omega, p)$ condition, that is

$$
t_{k+p}=t_{k}+\omega, \quad \gamma_{k+p}=\gamma_{k}+\omega \quad \text { and } \quad J_{k+p}=J_{k} .
$$

Remark 3.1. Note that $(\omega, p)$ condition is a discrete relation, which moves the interval $I_{k}$ into $I_{k+p}$. Then we have the following consequences.

(i) For any $\tau \in \mathbb{R}^{+}$, the interval $[\tau, \tau+\omega]$ can be decomposed as follows:

$$
\left[\tau, t_{i(\tau)+1}\right] \cup \bigcup_{j=i(\tau)+1}^{i(\tau)+p-1} I_{j} \cup\left[t_{i(\tau)+p}, \tau+\omega\right]
$$


(ii) For $t \in\left[t_{k}, t_{k+1}\right)$, we have (a) $t+\omega \in\left[t_{k+p}, t_{k+p+1}\right)$, (b) $\gamma(t)+\omega \in\left[t_{k+p}, t_{k+p+1}\right)$. Then,

$$
\gamma(t+\omega)=\gamma_{i(t+\omega)}=\gamma_{i(t)+p}=\gamma_{i(t)}+\omega=\gamma(t)+\omega
$$

For $\omega>0$, let $\mathrm{PC}_{\omega}$ be the set of all $n$-vector piecewise continuous function $x(t)$ with points of discontinuity of the first kind at $t=t_{i}, i \in \mathbb{N}$, periodic in $t$ of period $\omega$. Then $\left(\mathrm{PC}_{\omega},\|\cdot\|\right)$ is a Banach space with the supremum norm

$$
\|x\|=\max _{1 \leq i \leq n}\left\|x_{i}\right\|=\max _{1 \leq i \leq n}\left[\sup _{t \in \mathbb{R}^{+}}\left|x_{i}(t)\right|\right]=\max _{1 \leq i \leq n}\left[\sup _{t \in[\tau, \tau+\omega]}\left|x_{i}(t)\right|\right] .
$$

\subsection{Existence of periodic solutions}

In this subsection, we will establish the sufficient conditions for existence and uniqueness of the $\omega$-periodic solutions of the ICNN models with the IDEPCAG system (2.1a)-(2.1b).

Before giving our main result of this subsection, we need to establish some definitions and elementary facts.

Definition 3.2. For each $t, s \in[\tau, \tau+\omega]$, the Green's function for the ICNN models with the IDEPCAG system 2.1a -2.1b is given by $G(t, s)=\operatorname{diag}\left\{G_{i}(t, s)\right\}, i=1, \ldots, n$, where

$$
G_{i}(t, s)= \begin{cases}\left(\frac{\exp \left(\int_{\tau}^{\tau+\omega} a_{i}(u) d u\right)}{\exp \left(\int_{\tau}^{\tau+\omega} a_{i}(u) d u\right)-1}\right) \exp \left(\int_{t}^{s} a_{i}(\kappa) d \kappa\right), \quad \tau \leq s \leq t \leq \tau+\omega \\ \left(\frac{1}{\exp \left(\int_{\tau}^{\tau+\omega} a_{i}(u) d u\right)-1}\right) \exp \left(\int_{t}^{s} a_{i}(\kappa) d \kappa\right), \quad \tau \leq t<s \leq \tau+\omega\end{cases}
$$

Note that $G$ and $G_{i}$ are bi $\omega$-periodic, i.e., $G(t+\omega, s+\omega)=G(t, s)$ and the denominator in $G_{i}(t, s)$ for $i=1,2, \ldots, n$, is not zero since we have assumed that $a_{i}(c)>0$ for some $c \in[\tau, \tau+\omega]$. Observe that $G_{i}(t, s)$ has maximum and minimum values:

$$
\frac{1}{\exp \left(\int_{\tau}^{\tau+\omega} a_{i}(\kappa) d \kappa\right)-1} \leq G_{i}(t, s) \leq \frac{\exp \left(\int_{\tau}^{\tau+\omega} a_{i}(\kappa) d \kappa\right)}{\exp \left(\int_{\tau}^{\tau+\omega} a_{i}(\kappa) d \kappa\right)-1}, \quad t \leq s \leq t+\omega
$$

For the sake of convenience, we adopt the following notations

$$
c_{G}:=\max _{t, s \in[\tau, \tau+\omega]}|G(t, s)|=\max _{1 \leq i \leq n} \frac{\exp \left(\int_{\tau}^{\tau+\omega} a_{i}(\kappa) d \kappa\right)}{\exp \left(\int_{\tau}^{\tau+\omega} a_{i}(\kappa) d \kappa\right)-1} \quad \text { and } \quad a_{*}(t)=\min _{1 \leq i \leq n} a_{i}(t) .
$$

Using Definition 3.2, Remark 3.1 and bi w-periodicity of the Green's function, we have the following lemma. 
Lemma 3.3. Suppose that the condition $(\mathrm{P})$ holds and $x(t)$ is a solution of the ICNN models with the IDEPCAG system 2.1a) - 2.1b), then $x(t) \in \mathrm{PC}_{\omega}$ if and only if

$$
\begin{aligned}
x(t)= & \int_{\tau}^{\tau+\omega} G(t, s)[B(s) f(x(s))+C(s) g(x(\gamma(s)))+D(s)] d s \\
& +\sum_{k=i(\tau)+1}^{i(\tau)+p} G\left(t, t_{k}\right) \mathfrak{J}_{k}\left(x\left(t_{k}^{-}\right)\right),
\end{aligned}
$$

where $G(t, s)$ is the Green's function. In particular, we have

$$
\begin{aligned}
x_{i}(t)= & \int_{\tau}^{\tau+\omega} G_{i}(t, s)\left[\sum_{j=1}^{n} b_{i j}(s) f_{j}\left(x_{j}(s)\right)+\sum_{j=1}^{n} c_{i j}(s) g_{j}\left(x_{j}(\gamma(s))\right)+d_{i}(s)\right] d s \\
& +\sum_{k=i(\tau)+1}^{i(\tau)+p} G_{i}\left(t, t_{k}\right) \mathfrak{J}_{i k}\left(x_{i}\left(t_{k}^{-}\right)\right), \quad 1 \leq i \leq n .
\end{aligned}
$$

Proof. Necessity. Let $x(t) \in \mathrm{PC}_{\omega}$ be a solution of the ICNN models with the IDEPCAG system (2.1a)-2.1b), $\Phi(t)$ is a fundamental solution of $u^{\prime}(t)=-A(t) u(t)$ and $I_{n}=\operatorname{diag}(1,1, \ldots, 1)$. By Proposition 2.2, we have

$$
\begin{aligned}
x(t)= & \Phi(t, \tau) x_{0}+\int_{\tau}^{t} \Phi(t, s)[B(s) f(x(s))+C(s) g(x(\gamma(s)))+D(s)] d s \\
& +\sum_{k=i(\tau)+1}^{i(t)} \Phi\left(t, t_{k}\right) \mathfrak{J}_{k}\left(x\left(t_{k}^{-}\right)\right), \quad i(t)>i(\tau) .
\end{aligned}
$$

Since $x(\tau)=x_{0}=x(\tau+\omega)$, we get

$$
\begin{aligned}
x_{0}= & \left(I_{n}-\Phi(\tau+\omega, \tau)\right)^{-1} \int_{\tau}^{\tau+\omega} \Phi(\tau+\omega, s)[B(s) f(x(s))+C(s) g(x(\gamma(s)))+D(s)] d s \\
& +\sum_{k=i(\tau)+1}^{i(\tau)+p} \Phi\left(\tau+\omega, t_{k}\right) \mathfrak{J}_{k}\left(x\left(t_{k}^{-}\right)\right) .
\end{aligned}
$$

A substitution of 3.2 into 2.2 yields

$$
\begin{aligned}
x(t)=\Phi(t, \tau)\langle & \left(I_{n}-\Phi(\tau+\omega, \tau)\right)^{-1} \\
& \times \int_{\tau}^{\tau+\omega} \Phi(\tau+\omega, s)[B(s) f(x(s))+C(s) g(x(\gamma(s)))+D(s)] d s \\
& \left.+\sum_{k=i(\tau)+1}^{i(\tau)+p} \Phi\left(\tau+\omega, t_{k}\right) \mathfrak{J}_{k}\left(x\left(t_{k}^{-}\right)\right)\right\rangle
\end{aligned}
$$




$$
\begin{aligned}
& +\int_{\tau}^{t} \Phi(t, s)[B(s) f(x(s))+C(s) g(x(\gamma(s)))+D(s)] d s \\
& +\sum_{k=i(\tau)+1}^{i(t)} \Phi\left(t, t_{k}\right) \mathfrak{J}_{k}\left(x\left(t_{k}^{-}\right)\right) .
\end{aligned}
$$

Let $E=\left(\left(\Phi^{-1}(\tau) \Phi(\tau+\omega)\right)^{-1}-I_{n}\right)^{-1}=\operatorname{diag}\left\{\left(e^{\int_{\tau}^{\tau+\omega} a_{1}(s) d s}-1\right)^{-1}, \ldots,\left(e^{\int_{\tau}^{\tau+\omega}} a_{n}(s) d s-\right.\right.$ $\left.1)^{-1}\right\}$. Since $\left(I_{n}+E\right)=\left(\Phi^{-1}(\tau) \Phi(\tau+\omega)\right)^{-1} E=\left(I_{n}-\Phi^{-1}(\tau) \Phi(\tau+\omega)\right)^{-1}$, (3.3) becomes

$$
\begin{aligned}
x(t)= & \Phi(t, \tau)\left(I_{n}-\Phi(\tau+\omega, \tau)\right)^{-1} \\
& \times\left\langle\int_{\tau}^{t} \Phi(\tau+\omega, s)[B(s) f(x(s))+C(s) g(x(\gamma(s)))+D(s)] d s\right. \\
& \left.+\sum_{k=i(\tau)+1}^{i(\tau)+p} \Phi\left(\tau+\omega, t_{k}\right) \mathfrak{J}_{k}\left(x\left(t_{k}^{-}\right)\right)\right\rangle \\
& +\Phi(t, \tau)\left(I_{n}-\Phi(\tau+\omega, \tau)\right)^{-1} \\
& \times \int_{t}^{\tau+\omega} \Phi(\tau+\omega, s)[B(s) f(x(s))+C(s) g(x(\gamma(s)))+D(s)] d s \\
& +\int_{\tau}^{t} \Phi(t, s)[B(s) f(x(s))+C(s) g(x(\gamma(s)))+D(s)] d s+\sum_{k=i(\tau)+1}^{i(t)} \Phi\left(t, t_{k}\right) \mathfrak{J}_{k}\left(x\left(t_{k}^{-}\right)\right) \\
= & \int_{\tau}^{t} \Phi(t)\left(I_{n}+E\right) \Phi(s)^{-1}[B(s) f(x(s))+C(s) g(x(\gamma(s)))+D(s)] d s \\
& +\sum_{k=i(\tau)+1}^{i(\tau)+p} \Phi(t)\left(I_{n}+E\right) \Phi\left(t_{k}\right)^{-1} \mathfrak{J}_{k}\left(x\left(t_{k}^{-}\right)\right) \\
& +\int_{t}^{\tau+\omega} \Phi(t) E \Phi{ }^{-1}(s)[B(s) f(x(s))+C(s) g(x(\gamma(s)))+D(s)] d s \\
& +\sum_{k=i(\tau)+1}^{i(\tau)+p} \Phi(t) E \Phi\left(t_{k}\right)^{-1} \mathfrak{J}_{k}\left(x\left(t_{k}^{-}\right)\right) \\
& \int_{\tau}^{\tau+\omega} G(t, s)[B(s) f(x(s))+C(s) g(x(\gamma(s)))+D(s)] d s+\sum_{k=i(\tau)+1} G\left(t, t_{k}\right) \mathfrak{J}_{k}\left(x\left(t_{k}^{-}\right)\right) .
\end{aligned}
$$

This proves the Necessity.

Sufficiency. We will prove that if (3.1) has a unique solution, then $x(t) \in \mathrm{PC}_{\omega}$. Indeed, by the condition (P) and bi $\omega$-periodicity of the Green's function, $x(t+\omega)$ is a solution of (3.1):

$$
\begin{aligned}
& x(t+\omega) \\
= & \int_{\tau+\omega}^{\tau+2 \omega} G(t+\omega, s)[B(s) f(x(s))+C(s) g(x(\gamma(s)))+D(s)] d s
\end{aligned}
$$




$$
\begin{aligned}
& +\sum_{k=i(\tau)+p+1}^{i(\tau)+2 p} G\left(t+\omega, t_{k}\right) \mathfrak{J}_{k}\left(x\left(t_{k}^{-}\right)\right) \\
= & \int_{\tau}^{\tau+\omega} G(t+\omega, s+\omega)[B(s+\omega) f(x(s+\omega))+C(s+\omega) g(x(\gamma(s+\omega)))+D(s+\omega)] d s \\
& +\sum_{k=i(\tau)+1}^{i(\tau)+p} G\left(t+\omega, t_{k+p}\right) \mathfrak{J}_{k+p}\left(x\left(t_{k+p}^{-}\right)\right) \\
= & \int_{\tau}^{\tau+\omega} G(t, s)[B(s) f(x(s+\omega))+C(s) g(x(\gamma(s+\omega)))+D(s)] d s \\
& +\sum_{k=i(\tau)+1}^{i(\tau)+p} G\left(t, t_{k}\right) \mathfrak{J}_{k}\left(x\left(t_{k}^{-}\right)\right) .
\end{aligned}
$$

Then, $x(t+\omega)=x(t)$ and $x(t)$ is an $\omega$-periodic solution of the ICNN models with the IDEPCAG system 2.1a $-2.1 \mathrm{~b}$. This completes the proof of Lemma 3.3 .

Now, define the operator $\mathcal{T}: \mathrm{PC}_{\omega} \rightarrow \mathrm{PC}_{\omega}$ by

$$
(\mathcal{T} x)(t)=\left((\mathcal{T} x)_{1}(t),(\mathcal{T} x)_{2}(t), \ldots,(\mathcal{T} x)_{n}(t)\right)^{T},
$$

where

$$
\begin{aligned}
(\mathcal{T} x)_{i}(t)= & \int_{\tau}^{\tau+\omega} G_{i}(t, s)\left[\sum_{j=1}^{n} b_{i j}(s) f_{j}\left(x_{j}(s)\right)+\sum_{j=1}^{n} c_{i j}(s) g_{j}\left(x_{j}(\gamma(s))\right)+d_{i}(s)\right] d s \\
& +\sum_{k=i(\tau)+1}^{i(\tau)+p} G_{i}\left(t, t_{k}\right) \mathfrak{J}_{i k}\left(x_{i}\left(t_{k}^{-}\right)\right)
\end{aligned}
$$

for $i=1,2, \ldots, n$. By Lemma 3.3 , it is easy to verify that $x=x(t)$ is an $\omega$-periodic solution of the ICNN models with the IDEPCAG system (2.1a)-(2.1b) if and only if the operator $\mathcal{T}$ has one fixed point in $\mathrm{PC}_{\omega}$.

The following theorem is our main result of this subsection.

Theorem 3.4. If $\left(\mathrm{L}_{1}\right),\left(\mathrm{L}_{2}\right),(\mathrm{E})$ and $(\mathrm{P})$ are satisfied and

$$
c_{G} \cdot \max _{1 \leq i \leq n}\left\{\left[\int_{\tau}^{\tau+\omega}\left(\sum_{j=1}^{n} \mathfrak{L}_{j}^{f}\left|b_{i j}(s)\right|+\mathfrak{L}_{j}^{g}\left|c_{i j}(s)\right|\right) d s\right]+\sum_{k=i(\tau)+1}^{i(\tau)+p} \mathfrak{L}_{i k}^{J}\right\}<1 .
$$

Then the ICNN models with the IDEPCAG system (2.1a)-2.1b) has a unique w-periodic solution.

Proof. Define the operator $\mathcal{T}$ in $\mathrm{PC}_{\omega}$ by $\mathcal{T}: \mathrm{PC}_{\omega} \rightarrow \mathrm{PC}_{\omega}$ such that if $\varphi \in \mathrm{PC}_{\omega}$, then

$$
(\mathcal{T} \varphi)_{i}(t)=\int_{\tau}^{\tau+\omega} G_{i}(t, s)\left[\sum_{j=1}^{n} b_{i j}(s) f_{j}\left(\varphi_{j}(s)\right)+\sum_{j=1}^{n} c_{i j}(s) g_{j}\left(\varphi_{j}(\gamma(s))\right)+d_{i}(s)\right] d s
$$




$$
+\sum_{k=i(\tau)+1}^{i(\tau)+p} G_{i}\left(t, t_{k}\right) \mathfrak{J}_{i k}\left(\varphi_{i}\left(t_{k}^{-}\right)\right)
$$

for $i=1,2, \ldots, n$. The theorem follows if we prove that $\mathcal{T}$ has a fixed point. Let $\psi, \varphi$ be two functions in $\mathrm{PC}_{\omega}$. By using $\left(\mathrm{L}_{1}\right)$ and $\left(\mathrm{L}_{2}\right)$, we can deduce

$$
\begin{aligned}
& \left\|(\mathcal{T} \psi)_{i}(\cdot)-(\mathcal{T} \varphi)_{i}(\cdot)\right\|=\max _{i \in[1, ., n]}\left\{\max _{t \in[\tau, \tau+\omega]}\left|(\mathcal{T} \psi)_{i}(t)-(\mathcal{T} \varphi)_{i}(t)\right|\right\} \\
& \leq \max _{i \in[1, ., n]}\left\{\int _ { \tau } ^ { \tau + \omega } \operatorname { m a x } _ { t \in [ \tau , \tau + \omega ] } | G _ { i } ( t , s ) | \left[\sum_{j=1}^{n}\left|b_{i j}(s)\right|\left|f_{j}\left(\psi_{j}(s)\right)-f_{j}\left(\varphi_{j}(s)\right)\right|\right.\right. \\
& \left.+\sum_{j=1}^{n}\left|c_{i j}(s)\right|\left|g_{j}\left(\psi_{j}(\gamma(s))\right)-g_{j}\left(\varphi_{j}(\gamma(s))\right)\right|\right] d s \\
& \left.+\sum_{k=i(\tau)+1}^{i(\tau)+p} \max _{t \in[\tau, \tau+\omega]}\left|G_{i}\left(t, t_{k}\right)\right|\left|\mathfrak{J}_{i k}\left(\psi_{i}\left(t_{k}^{-}\right)\right)-\mathfrak{J}_{i k}\left(\varphi_{i}\left(t_{k}^{-}\right)\right)\right|\right\} \\
& \leq \max _{i \in[1, ., n]}\left\{\int _ { \tau } ^ { \tau + \omega } \operatorname { m a x } _ { t \in [ \tau , \tau + \omega ] } | G _ { i } ( t , s ) | \left[\sum_{j=1}^{n}\left|b_{i j}(s)\right| \mathfrak{L}_{j}^{f}\left|\psi_{j}(s)-\varphi_{j}(s)\right|\right.\right. \\
& \left.+\sum_{j=1}^{n}\left|c_{i j}(s)\right| \mathfrak{L}_{j}^{g}\left|\psi_{j}(\gamma(s))-\varphi_{j}(\gamma(s))\right|\right] d s \\
& \left.+\sum_{k=i(\tau)+1}^{i(\tau)+p} \max _{t \in[\tau, \tau+\omega]}\left|G_{i}(t, s)\right| \mathfrak{L}_{i k}^{J}\left|\psi_{i}\left(t_{k}^{-}\right)-\varphi_{i}\left(t_{k}^{-}\right)\right|\right\} \\
& \leq c_{G} \max _{i \in[1, ., n]}\left\{\int_{\tau}^{\tau+\omega}\left[\sum_{j=1}^{n} \mathfrak{L}_{j}^{f}\left|b_{i j}(s)\right|+\sum_{j=1}^{n} \mathfrak{L}_{j}^{g}\left|c_{i j}(s)\right|\right] d s+\sum_{k=i(\tau)+1}^{i(\tau)+p} \mathfrak{L}_{i k}^{J}\right\}\|\psi-\varphi\| .
\end{aligned}
$$

Finally, (3.5) implies

$$
\begin{aligned}
& \|\mathcal{T} \psi-\mathcal{T} \varphi\| \\
\leq & c_{G} \cdot \max _{1 \leq i \leq n}\left\{\left[\int_{\tau}^{\tau+\omega}\left(\sum_{j=1}^{n} \mathfrak{L}_{j}^{f}\left|b_{i j}(s)\right|+\mathfrak{L}_{j}^{g}\left|c_{i j}(s)\right|\right) d s\right]+\sum_{k=i(\tau)+1}^{i(\tau)+p} \mathfrak{L}_{i k}^{J}\right\}\|\psi-\varphi\| .
\end{aligned}
$$

From (3.4), the mapping $\mathcal{T}$ is a contraction. Hence, the mapping $\mathcal{T}$ possesses a unique fixed point $\varphi^{*} \in \mathrm{PC}_{\omega}, \mathcal{T} \varphi^{*}=\varphi^{*}$. By Lemma 3.3. $\varphi^{*}$ is the unique $\omega$-periodic solution of the ICNN models with the IDEPCAG system $(2.1 \mathrm{a})-(2.1 \mathrm{~b})$ in $\mathrm{PC}_{\omega}$. The proof of Theorem 3.4 is completed.

Remark 3.5. In our main results, the condition 3.4 is very important which guarantees the operator $\mathcal{T}$ mapping $\mathrm{PC}_{\omega}$ into $\mathrm{PC}_{\omega}$. Moreover, in the case of the DEPCAG 
system 2.18 without impulsive effects, the impulsive condition can be omitted and Theorem 3.4 is always valid.

The obtained results are different from and less restrictive than those given in 1 , Lemma 4.1] and [1, Theorem 4.1], and the assumptions (C3), (C4) and (C7) in [1] are relaxed. See Example 4.2 .

When considering CNN models (2.18) without impulsive effects, our results lead to the ones in 17. Moreover, our results can be extend to CNN models with piecewise constant argument [15, 17].

From Theorem 3.4, we can derive the following results.

Corollary 3.6. If $\left(\mathrm{L}_{1}\right),\left(\mathrm{L}_{2}\right),(\mathrm{P})$ and 2.15$)$ are satisfied and

$$
\max _{1 \leq i \leq n}\left\{\frac{\exp \left(\bar{a}_{i} \omega\right)}{\exp \left(\underline{a}_{i} \omega\right)-1}\left[\sum_{j=1}^{n}\left(\mathfrak{L}_{j}^{f}\left|\bar{b}_{i j}\right|+\mathfrak{L}_{j}^{g}\left|\bar{c}_{i j}\right|\right) \omega+\sum_{k=i(\tau)+1}^{i(\tau)+p} \mathfrak{L}_{i k}^{J}\right]\right\}<1,
$$

where, $\bar{a}_{i}=\sup _{t \in \mathbb{R}^{+}} a_{i}(t), \underline{a}_{i}=\inf _{t \in \mathbb{R}^{+}} a_{i}(t)>0, \bar{b}_{i j}=\sup _{t \in \mathbb{R}^{+}}\left|b_{i j}(t)\right|$ and $\bar{c}_{i j}=$ $\sup _{t \in \mathbb{R}^{+}}\left|c_{i j}(t)\right|$. Then the ICNN models with the IDEPCAG system (2.1a) 2.1b) has a unique $\omega$-periodic solution.

Corollary 3.7. For $a_{i}(t) \equiv a_{i}>0, b_{i j}(t) \equiv b_{i j}$ and $c_{i j}(t) \equiv c_{i j}$ constants, if $\left(\mathrm{L}_{1}\right),\left(\mathrm{L}_{2}\right)$, (P) and 2.16) are satisfied and

$$
\max _{1 \leq i \leq n}\left\{\frac{\exp \left(a_{i} \omega\right)}{\exp \left(a_{i} \omega\right)-1}\left[\sum_{j=1}^{n}\left(\mathfrak{L}_{j}^{f}\left|b_{i j}\right|+\mathfrak{L}_{j}^{g}\left|c_{i j}\right|\right) \omega+\sum_{k=i(\tau)+1}^{i(\tau)+p} \mathfrak{L}_{i k}^{J}\right]\right\}<1 .
$$

Then the ICNN models with the IDEPCAG system 2.1a)-2.1b), but with the respective constant coefficients, has a unique $\omega$-periodic solution.

Applying our results to ICNN models with the IDEGPCD system (2.17a)-2.17b), we can reduce the following results.

Corollary 3.8. If $\left(\mathrm{L}_{1}\right),\left(\mathrm{L}_{2}\right),\left(\mathrm{E}^{\prime}\right),(\mathrm{P})$ and (3.4) are satisfied. Then the ICNN models with the IDEGPCD system (2.17a) - 2.17b) has a unique w-periodic solution.

Corollary 3.9. If $\left(\mathrm{L}_{1}\right),\left(\mathrm{L}_{2}\right),\left(\mathrm{E}^{\prime}\right),(\mathrm{P})$ and (3.7) are satisfied. Then the ICNN models with the IDEGPCD system (2.17a) - 2.17b), but with the respective constant coefficients, has a unique $\omega$-periodic solution.

Applying our results to CNN models with DEGPCD system 2.18 without impulsive effects, we have 
Corollary 3.10. If $\left(\mathrm{L}_{1}\right),(\mathrm{P}), 2.15$ and

$$
\max _{1 \leq i \leq n}\left\{\frac{\exp \left(\bar{a}_{i} \omega\right)}{\exp \left(\underline{a}_{i} \omega\right)-1}\left[\sum_{j=1}^{n}\left(\mathfrak{L}_{j}^{f}\left|\bar{b}_{i j}\right|+\mathfrak{L}_{j}^{g}\left|\bar{c}_{i j}\right|\right) \omega\right]\right\}<1
$$

are satisfied, where $\bar{a}_{i}=\sup _{t \in \mathbb{R}^{+}} a_{i}(t), \underline{a}_{i}=\inf _{t \in \mathbb{R}^{+}} a_{i}(t)>0, \bar{b}_{i j}=\sup _{t \in \mathbb{R}^{+}}\left|b_{i j}(t)\right|$ and $\bar{c}_{i j}=\sup _{t \in \mathbb{R}^{+}}\left|c_{i j}(t)\right|$. Then the CNN models with the DEPCAG system (2.18) has a unique $\omega$-periodic solution.

Corollary 3.11. For $a_{i}(t) \equiv a_{i}>0, b_{i j}(t) \equiv b_{i j}$ and $c_{i j}(t) \equiv c_{i j}$ constants, if $\left(\mathrm{L}_{1}\right),(\mathrm{P})$, 2.16 and

$$
\max _{1 \leq i \leq n}\left\{\frac{\exp \left(a_{i} \omega\right)}{\exp \left(a_{i} \omega\right)-1}\left[\sum_{j=1}^{n}\left(\mathfrak{L}_{j}^{f}\left|b_{i j}\right|+\mathfrak{L}_{j}^{g}\left|c_{i j}\right|\right) \omega\right]\right\}<1
$$

are satisfied. Then the CNN models with the DEPCAG system 2.18, but with the respective constant coefficients, has a unique $\omega$-periodic solution.

Corollary 3.12. If $\left(\mathrm{L}_{1}\right),\left(\mathrm{E}^{\prime}\right),(\mathrm{P})$ and (3.8) are satisfied. Then the CNN models with the DEGPCD system 2.17a has a unique $\omega$-periodic solution.

Corollary 3.13. If $\left(\mathrm{L}_{1}\right),\left(\mathrm{E}^{\prime}\right),(\mathrm{P})$ and $(3.9)$ are satisfied. Then the CNN models with the DEGPCD system 2.17a), with the respective constant coefficients, has a unique w-periodic solution.

Remark 3.14. Theorem 3.4 reduces to the results of $\left[10\right.$, Theorem 4.1] with $\gamma(t)=2\left[\frac{t+1}{2}\right]$, Corollary 3.10 reduces to the results of [17, Corollary 1] without impulsive effects and Corollary 3.12 reduces to the results of [15. Corollary 3] with generalized piecewise constant delay. It is shown that our results are general and they complement the previously known results.

\subsection{Global exponential stability of the periodic solution}

The following result will obtain sufficient conditions for the global exponential stability of the $\omega$-periodic solution of the ICNN models with IDEGPCD system $(2.1 \mathrm{a})-(2.1 \mathrm{~b})$.

The following notations are required in the section:

$$
\begin{gathered}
a_{*}(t)=\min _{1 \leq i \leq n} a_{i}(t), \quad \underline{a}_{*}=\inf _{t \in \mathbb{R}^{+}} a_{*}(t), \quad \bar{b}_{i j}=\sup _{t \in \mathbb{R}^{+}}\left|b_{i j}(t)\right|, \quad \bar{c}_{i j}=\sup _{t \in \mathbb{R}^{+}}\left|c_{i j}(t)\right|, \\
\vartheta^{-}=\sup _{\kappa \in \mathbb{N}}\left(t_{\kappa+1}-\gamma_{\kappa}\right), \quad \vartheta^{+}=\sup _{\kappa \in \mathbb{N}}\left(\gamma_{\kappa}-t_{\kappa}\right), \quad \vartheta_{\kappa}=t_{\kappa+1}-t_{\kappa}, \quad \vartheta=\sup _{\kappa \in \mathbb{N}} \vartheta_{\kappa}, \\
\mathfrak{L}_{\kappa}^{J}=\max _{1 \leq i \leq n} \mathfrak{L}_{i \kappa}^{J}, \quad \mathfrak{L}_{i(t)}=\max _{i(\tau)+1 \leq \kappa \leq i(t)} \frac{\ln \left(1+\mathfrak{L}_{\kappa}^{J}\right)}{\vartheta_{\kappa}}, \quad \mu^{*}(t)=\max _{1 \leq i \leq n} \mu_{i}(t),
\end{gathered}
$$


and

$$
\mu_{i}(t)=\sum_{j=1}^{n} \mathfrak{L}_{j}^{f}\left|b_{i j}(t)\right|+\frac{1}{1-\widehat{\nu}} \sum_{j=1}^{n} \mathfrak{L}_{j}^{g}\left|c_{i j}(t)\right| \exp \left(\int_{\gamma(t)}^{t} a_{*}(s) d s\right),
$$

where

$$
\max _{1 \leq i \leq n}\left(\sup _{k \in \mathbb{N}} \int_{t_{k}}^{\gamma_{k}}\left[\sum_{j=1}^{n} \mathfrak{L}_{j}^{f}\left|b_{i j}(s)\right|+\sum_{j=1}^{n} \mathfrak{L}_{j}^{g}\left|c_{i j}(s)\right| \exp \left(\int_{\gamma_{k}}^{s} a_{*}(s) d s\right)\right]\right) \leq \widehat{\nu}<1 .
$$

Here we assume the stability condition.

(S) Stability condition. There exists $\rho \in \mathbb{R}^{+}$, such that

$$
a_{*}(t)-\mu^{*}(t)-\mathfrak{L}_{i(t)} \geq \rho>0, \quad t \in \mathbb{R}^{+} .
$$

Theorem 3.15. If $\left(\mathrm{L}_{1}\right),\left(\mathrm{L}_{2}\right),(\mathrm{E}),(\mathrm{P}),(\mathrm{S})$ and $(3.4)$ are satisfied. Then the $\omega$-periodic solution of the ICNN models with the IDEPCAG system (2.1a)-2.1b is globally exponentially stable.

To prove Theorem 3.15, we need the following lemma.

Lemma 3.16. If $\left(\mathrm{L}_{1}\right),\left(\mathrm{L}_{2}\right)$ and $(\mathrm{E})$ are satisfied, then the solutions $\varphi$ and $\psi$ of the the IDEPCAG system 2.1a) 2.1b satisfy for all $t \geq \tau$ the inequality

$$
|\varphi(t)-\psi(t)| \leq|\varphi(\tau)-\psi(\tau)| \exp \left(-\int_{\tau}^{t} \lambda(s) d s\right)
$$

where $\lambda(t)=a_{*}(t)-\mu^{*}(t)-\mathfrak{L}_{i(t)}$.

Proof. Suppose that $\varphi(t)=\left(\varphi_{1}, \ldots, \varphi_{n}\right)^{T}$ and $\psi(t)=\left(\psi_{1}, \ldots, \psi_{n}\right)^{T}$ are arbitrary solutions of the IDEPCAG system 2.1a $-2.1 \mathrm{~b}$. Let $y(t)=\varphi(t)-\psi(t)$ and by (2.1a) $-2.1 \mathrm{~b}$ it follows that $y(t)$ satisfies

$$
\begin{aligned}
\dot{y}(t)= & -A(t) y(t)+B(t)[f(y(t)+\psi(t))-f(\psi(t))] \\
& +C(t)[g(y(\gamma(t))+\psi(\gamma(t)))-g(\psi(\gamma(t)))], \\
\left.\Delta y\right|_{t=t_{k}}= & J_{k}\left(y\left(t_{k}^{-}\right)+\psi\left(t_{k}^{-}\right)\right)-J_{k}\left(\psi\left(t_{k}^{-}\right)\right), \quad k \in \mathbb{N} .
\end{aligned}
$$

By Proposition 2.2, it can be proved that

$$
\begin{aligned}
y(t)= & \Phi(t, \tau) y(\tau)+\int_{\tau}^{t} \Phi(t, s) \mathcal{R}(s, y(s)) d s \\
& +\sum_{k=i(\tau)+1}^{i(t)} \Phi\left(t, t_{k}\right) \mathcal{J}_{k}\left(y\left(t_{k}^{-}\right)\right), \quad i(t)>i(\tau),
\end{aligned}
$$


where

$$
\mathcal{R}(s, y(s)):=B(s)[f(y(s)+\psi(s))-f(\psi(s))]+C(s)[g(y(\beta(s))+\psi(\gamma(s)))-g(\psi(\gamma(s)))],
$$

and

$$
\mathcal{J}_{k}\left(y\left(t_{k}^{-}\right)\right):=\mathfrak{J}_{k}\left(y\left(t_{k}^{-}\right)+\psi\left(t_{k}^{-}\right)\right)-\mathfrak{J}_{k}\left(\psi\left(t_{k}^{-}\right)\right) .
$$

Notice that $\left(\mathrm{L}_{1}\right)$ and $\left(\mathrm{L}_{2}\right)$ imply that

$$
\begin{aligned}
& \left|\mathcal{R}_{i}(s, y(s))\right| \leq\left(\sum_{j=1}^{n} \mathfrak{L}_{j}^{f}\left|b_{i j}(s)\right|\left|y_{j}(s)\right|+\sum_{j=1}^{n} \mathfrak{L}_{j}^{g}\left|c_{i j}(s)\right|\left|y_{j}(\gamma(s))\right|\right) \\
& |\mathcal{R}(s, y(s))| \leq \max _{1 \leq i \leq n}\left(\sum_{j=1}^{n} \mathfrak{L}_{j}^{f}\left|b_{i j}(s)\right||y(s)|+\sum_{j=1}^{n} \mathfrak{L}_{j}^{g}\left|c_{i j}(s)\right||y(\gamma(s))|\right)
\end{aligned}
$$

and

$$
\left|\mathcal{J}_{k}\left(y\left(t_{k}^{-}\right)\right)\right| \leq \mathfrak{L}_{k}^{J}\left|y\left(t_{k}^{-}\right)\right|
$$

By $(3.12)$, we can deduce that $v_{i}(t)=\exp \left(\int_{\tau}^{t} a_{*}(s) d s\right)\left|y_{i}(t)\right|$ satisfies

$$
\begin{aligned}
\left|v_{i}(t)\right| \leq & \left|\varphi_{i}(\tau)-\psi_{i}(\tau)\right| \\
& +\int_{\tau}^{t}\left[\sum_{j=1}^{n} \mathfrak{L}_{j}^{f}\left|b_{i j}(s)\right|\left|v_{j}(s)\right|+\sum_{j=1}^{n} \mathfrak{L}_{j}^{g}\left|c_{i j}(s)\right|\left|v_{j}(\gamma(s))\right| \exp \left(\int_{\gamma(s)}^{s} a_{*}(\kappa) d \kappa\right)\right] d s \\
& +\sum_{k=i(\tau)+1}^{i(t)} \mathfrak{L}_{k}^{J}\left|v_{i}\left(t_{k}^{-}\right)\right|,
\end{aligned}
$$

or

$$
\begin{aligned}
|v(t)| \leq & |\varphi(\tau)-\psi(\tau)| \\
& +\max _{1 \leq i \leq n} \int_{\tau}^{t}\left[\sum_{j=1}^{n} \mathfrak{L}_{j}^{f}\left|b_{i j}(s)\right||v(s)|+\sum_{j=1}^{n} \mathfrak{L}_{j}^{g}\left|c_{i j}(s)\right||v(\gamma(s))| \exp \left(\int_{\gamma(s)}^{s} a_{*}(\kappa) d \kappa\right)\right] d s \\
& +\sum_{k=i(\tau)+1}^{i(t)} \mathfrak{L}_{k}^{J}\left|v\left(t_{k}^{-}\right)\right|
\end{aligned}
$$

for $i(t)>i(\tau)$.

Hence, by Lemma 2.3 of the IDEPCAG's Gronwall inequality implies

$$
\begin{aligned}
|v(t)| \leq & |\varphi(\tau)-\psi(\tau)| \prod_{k=i(\tau)+1}^{i(t)}\left(1+\mathfrak{L}_{k}^{J}\right) \\
& \times \exp \left\{\max _{1 \leq i \leq n} \int_{\tau}^{t} \sum_{j=1}^{n} \mathfrak{L}_{j}^{f}\left|b_{i j}(s)\right|+\frac{1}{1-\widehat{v}} \sum_{j=1}^{n} \mathfrak{L}_{j}^{g}\left|c_{i j}(s)\right| \exp \left(\int_{\gamma(s)}^{s} a_{*}(\kappa) d \kappa\right)\right\} .
\end{aligned}
$$


Then, we obtain

$$
|\varphi(t)-\psi(t)| \leq|\varphi(\tau)-\psi(\tau)| \prod_{k=i(\tau)+1}^{i(t)}\left(1+\mathfrak{L}_{k}^{J}\right) \exp \left(-\int_{\tau}^{t} a_{*}(s) d s+\max _{1 \leq i \leq n} \int_{\tau}^{t} \mu_{i}(s) d s\right)
$$

or

$$
\begin{aligned}
& |\varphi(t)-\psi(t)| \\
\leq & |\varphi(\tau)-\psi(\tau)| \exp \left\{\int_{\tau}^{t}\left(-a_{*}(s)+\max _{1 \leq i \leq n} \mu_{i}(s)+\max _{i(\tau)+1 \leq k \leq i(t)} \frac{\ln \left(1+\mathfrak{L}_{k}^{J}\right)}{\vartheta_{k}}\right) d s\right\},
\end{aligned}
$$

and the statement 3.11 follows.

Proof of Theorem 3.15. According to Theorem 3.4, we know that the ICNN models with the IDEPCAG system 2.1a $-2.1 \mathrm{~b}$ has an $\omega$-periodic solution $x^{*}(t)=\left(x_{1}^{*}(t), \ldots, x_{n}^{*}(t)\right)^{T}$ with initial value $x^{*}(\tau)=\left(x_{1}^{*}(\tau), \ldots, x_{n}^{*}(\tau)\right)^{T}$.

Suppose that $x(t)=\left(x_{1}(t), \ldots, x_{n}(t)\right)^{T}$ is an arbitrary solution of the ICNN models with the IDEPCAG system 2.1a) -2.1b with initial value $x(\tau)=\left(x_{1}(\tau), \ldots, x_{n}(\tau)\right)^{T}$. Consider the change of variables

$$
z(t)=x(t)-x^{*}(t)=\left(x_{1}(t)-x_{1}^{*}(t), \ldots, x_{n}(t)-x_{n}^{*}(t)\right)^{T} .
$$

Then it follows from system $2.1 \mathrm{a}-2.1 \mathrm{~b}$ ) that

$$
\begin{aligned}
z_{i}^{\prime}(t) & =-a_{i}(t) z_{i}(t)+\sum_{j=1}^{n} b_{i j}(t) \widetilde{f}_{j}\left(z_{j}(t)\right)+\sum_{j=1}^{n} c_{i j}(t) \widetilde{g}_{j}\left(z_{j}(\gamma(s))\right), \quad t \neq t_{k}, \\
\left.\Delta z_{i}\right|_{t=t_{k}} & =\widetilde{\mathfrak{J}}_{i k}\left(z_{i}\left(t_{k}^{-}\right)\right), \quad i=1,2, \ldots, n, k \in \mathbb{N},
\end{aligned}
$$

where

$$
\begin{aligned}
\tilde{f}_{j}\left(z_{j}(t)\right) & =f_{j}\left(z_{j}(t)+x_{j}^{*}(t)\right)-f_{j}\left(x_{j}^{*}(t)\right), \\
\widetilde{g}_{j}\left(z_{j}(\gamma(t))\right) & =g_{j}\left(z_{j}(\gamma(t))+x_{j}^{*}(\gamma(t))\right)-g_{j}\left(x_{j}^{*}(\gamma(t))\right), \\
\widetilde{\mathfrak{J}}_{i k}\left(z_{i}\left(t_{k}^{-}\right)\right) & =\mathfrak{J}_{k}\left(z_{i}\left(t_{k}^{-}\right)+x_{i}^{*}\left(t_{k}^{-}\right)\right)-\mathfrak{J}_{k}\left(x_{i}^{*}\left(t_{k}^{-}\right)\right) .
\end{aligned}
$$

Now, by Proposition 2.2, it can be proved that

$$
\begin{aligned}
z_{i}(t)= & e^{-\int_{\tau}^{t} a_{i}(s) d s} z_{i}(\tau) \\
& +\int_{\tau}^{t} e^{-\int_{s}^{t} a_{i}(u) d u}\left[\sum_{j=1}^{n} b_{i j}(s) \widetilde{f}_{j}\left(z_{j}(s)\right)+\sum_{j=1}^{n} c_{i j}(s) \widetilde{g}_{j}\left(z_{j}(\gamma(s))\right)\right] d s \\
& +\sum_{k=i(\tau)+1}^{i(t)} e^{-\int_{t_{k}}^{t} a(s) d s} \widetilde{\mathfrak{J}}_{i k}\left(z_{i}\left(t_{k}^{-}\right)\right), \quad i(t)>i(\tau),
\end{aligned}
$$


and similar to the proof of Lemma 3.16, we obtain

$$
\max _{1 \leq i \leq n}\left|z_{i}(t)\right| \leq \max _{1 \leq i \leq n}\left|z_{i}(\tau)\right| \exp \left(-\int_{\tau}^{t}\left[a_{*}(s)-\mu^{*}(s)-\mathfrak{L}_{i(s)}\right] d s\right) .
$$

Thus, from the assumption of (3.10), we can conclude that the $\omega$-periodic solution of the ICNN models with the IDEPCAG system 2.1a)-(2.1b) is globally exponentially stable and this completes the proof of Theorem 3.15 .

Remark 3.17. Different from the methods used in [1, 2], the relationship that $\|y(\beta(t))\| \leq$ $\bar{B}\|y(t)\|$, where $\bar{B}=\left\{1-\bar{\theta}\left[\alpha_{2}+\alpha_{3}\left(1+\bar{\theta} \alpha_{2}\right) e^{\bar{\theta} \alpha_{3}}\right]\right\}^{-1}>0$ in 1, Lemma 3.1] and $\bar{B}=$ $\left\{1-\left[k_{2} \bar{\theta}+\left(k_{1} \bar{\theta}+l p\right)\left(1+k_{2} \bar{\theta}\right)(1+l)^{p} e^{k_{1} \bar{\theta}}\right]\right\}^{-1}$ in 2 , Lemma 5.2.5] is not required in the present paper. Because this relationship is not necessary for the proposed technique of IDEPCAG's Gronwall inequality here.

Remark 3.18. The stability criteria in $\sqrt{1}$ are depended on the upper and lower bounds $\bar{\theta}$ and $\underline{\theta}$. It requires that $\gamma-\alpha_{1}-\bar{B} \alpha_{2}-\frac{\ln (1+l)}{\underline{\theta}}>0$ in 1 , Theorem 3.1]. Thus, those results cannot be used to obtain the stability of neural networks for any $\bar{\theta}\left[\alpha_{2}+\alpha_{3}\left(1+\bar{\theta} \alpha_{2}\right) e^{\bar{\theta} \alpha_{3}}\right]>$ 1. Then, we can choose proper parameter which the stability criteria in [1] are not satisfied. Hence, our results can be applied more convenient than the results in 1 .

Remark 3.19. The existence criterion (E) and the stability criterion (S) can be easily solved by using some existing software, for example, the MATLAB.

Remark 3.20. Theorem 3.15 reduces to the stability result of [10, Theorem 4.2] with the classic piecewise alternately advanced and retarded argument, [14, Theorem 4.4] and [1, Theorem 4.2] with generalized piecewise constant delay.

It should be pointed that, because of the complexity of the results, the problem of finding appropriate parameters is a difficult task. Therefore, in order to easily check the applicability of the results, we will give corollaries as follows.

Corollary 3.21. If the assumptions $\left(\mathrm{L}_{1}\right),\left(\mathrm{L}_{2}\right), 2.15$ are satisfied and

$$
\underline{a}_{*}>\max _{1 \leq i \leq n}\left(\sum_{j=1}^{n} \mathfrak{L}_{j}^{f}\left|\bar{b}_{i j}\right|+\sum_{j=1}^{n} \mathfrak{L}_{j}^{g}\left|\bar{c}_{i j}\right| \frac{e^{\bar{a}_{*} \cdot \vartheta^{-}}}{1-\widehat{\nu}}\right)+\sup _{\kappa \in \mathbb{N}} \frac{\ln \left(1+\mathfrak{L}_{\kappa}^{J}\right)}{\vartheta_{\kappa}}
$$

where

$$
\sup _{k \in \mathbb{N}}\left[\max _{1 \leq i \leq n}\left(\sum_{j=1}^{n} \mathfrak{L}_{j}^{f}\left|\bar{b}_{i j}\right|+\sum_{j=1}^{n} \mathfrak{L}_{j}^{g}\left|\bar{c}_{i j}\right|\left(\frac{1-e^{-\underline{a}_{*} \cdot \vartheta_{k}^{+}}}{\underline{a}_{*}}\right)\right) \cdot \vartheta_{k}^{+}\right] \leq \widehat{\nu}<1 .
$$

Then the ICNN models with the IDEPCAG system 2.1a) 2.1b is globally exponentially stable. 
Corollary 3.22. If the assumptions of Corollary 3.6, (3.13) and (3.14) are satisfied. Then the $\omega$-periodic solution of the ICNN models with the IDEPCAG system (2.1a) $-(2.1 \mathrm{~b})$ is globally exponentially stable.

Corollary 3.23. If the assumptions of Corollary 3.7 are satisfied and

$$
a_{*}>\max _{1 \leq i \leq n}\left(\sum_{j=1}^{n} \mathfrak{L}_{j}^{f}\left|b_{i j}\right|+\sum_{j=1}^{n} \mathfrak{L}_{j}^{g}\left|c_{i j}\right| \frac{e^{a_{*} \cdot \vartheta^{-}}}{1-\bar{\nu}}\right)+\sup _{\kappa \in \mathbb{N}} \frac{\ln \left(1+\mathfrak{L}_{\kappa}^{J}\right)}{\vartheta_{\kappa}}
$$

where $a_{*}=\inf _{1 \leq i \leq n} a_{i}$ and

$$
\sup _{k \in \mathbb{N}}\left[\max _{1 \leq i \leq n}\left(\sum_{j=1}^{n} \mathfrak{L}_{j}^{f}\left|b_{i j}\right|+\sum_{j=1}^{n} \mathfrak{L}_{j}^{g}\left|c_{i j}\right| \frac{1-e^{-a_{*} \cdot \vartheta_{k}^{+}}}{a_{*}}\right) \cdot \vartheta_{k}^{+}\right] \leq \bar{\nu}<1 .
$$

Then the $\omega$-periodic solution of the ICNN models with the IDEPCAG system (2.1a) - 2.1b) (the respective constant coefficients) is globally exponentially stable.

Note that if we consider the deviation argument that is of the constant delay of generalized type, i.e., $\gamma(t)=\gamma_{i}=t_{i}$, if $t \in\left[t_{i}, t_{i+1}\right), i \in \mathbb{N}$. We can easily to see that $\vartheta^{+}=0$ and $\vartheta^{-}=\vartheta$. Then we have the following corollary.

Corollary 3.24. If the assumptions of Corollary 3.8 are satisfied and

$$
\underline{a}_{*}>\max _{1 \leq i \leq n} \sum_{j=1}^{n}\left(\mathfrak{L}_{j}^{f}\left|\bar{b}_{i j}\right|+\mathfrak{L}_{j}^{g}\left|\bar{c}_{i j}\right| \exp \left(\bar{a}_{*} \cdot \vartheta\right)\right)+\sup _{\kappa \in \mathbb{N}} \frac{\ln \left(1+\mathfrak{L}_{\kappa}^{J}\right)}{\vartheta_{\kappa}} .
$$

Then the w-periodic solution of the ICNN models with the IDEGPCD system (2.17a)(2.17b) is globally exponentially stable.

Remark 3.25. Corollary 3.24 reduces to the results of [14, Theorem 4.4].

Corollary 3.26. If the assumptions of Corollary 3.9 are satisfied and

$$
a_{*}>\max _{1 \leq i \leq n} \sum_{j=1}^{n}\left(\mathfrak{L}_{j}^{f}\left|b_{i j}\right|+\mathfrak{L}_{j}^{g}\left|c_{i j}\right| \exp \left(a_{*} \cdot \vartheta\right)\right)+\sup _{\kappa \in \mathbb{N}} \frac{\ln \left(1+\mathfrak{L}_{\kappa}^{J}\right)}{\vartheta_{\kappa}} .
$$

Then the $\omega$-periodic solution of the IDEGPCD system 2.17a)-2.17b) (the respective constant coefficients) is globally exponentially stable.

Remark 3.27. Corollary 3.26 generalizes corresponding result obtained by [1, Theorem 4.2] and [2, Theorem 7.2.1] under complicated and stronger conditions.

As a direct consequence of the method, we have the following result without impulsive effects. 
Corollary 3.28. If the assumptions of Corollary 3.10 and (3.14) are satisfied and

$$
\underline{a}_{*}>\max _{1 \leq i \leq n}\left(\sum_{j=1}^{n} \mathfrak{L}_{j}^{f}\left|\bar{b}_{i j}\right|+\sum_{j=1}^{n} \mathfrak{L}_{j}^{g}\left|\bar{c}_{i j}\right| \frac{e^{\bar{a}_{*} \cdot \vartheta^{-}}}{1-\widehat{\nu}}\right) .
$$

Then the $\omega$-periodic solution of the CNN models with the DEPCAG system (2.18) is globally exponentially stable.

Corollary 3.29. If the assumptions of Corollary 3.11 and (3.16) are satisfied and

$$
a_{*}>\max _{1 \leq i \leq n}\left(\sum_{j=1}^{n} \mathfrak{L}_{j}^{f}\left|b_{i j}\right|+\sum_{j=1}^{n} \mathfrak{L}_{j}^{g}\left|c_{i j}\right| \frac{e^{a_{*} \cdot \vartheta^{-}}}{1-\bar{\nu}}\right) .
$$

Then the $\omega$-periodic solution of the CNN models with the DEPCAG system (2.18), but with the respective constant coefficients, is globally exponentially stable.

Corollary 3.30. If the assumptions of Corollary 3.12 are satisfied and

$$
\underline{a}_{*}>\max _{1 \leq i \leq n} \sum_{j=1}^{n}\left(\mathfrak{L}_{j}^{f}\left|\bar{b}_{i j}\right|+\mathfrak{L}_{j}^{g}\left|\bar{c}_{i j}\right| \exp \left(\bar{a}_{*} \cdot \vartheta\right)\right) .
$$

Then the w-periodic solution of the CNN models with the DEGPCD system (2.17a) is globally exponentially stable.

Corollary 3.31. If the assumptions of Corollary 3.13 are satisfied and

$$
a_{*}>\max _{1 \leq i \leq n} \sum_{j=1}^{n}\left(\mathfrak{L}_{j}^{f}\left|b_{i j}\right|+\mathfrak{L}_{j}^{g}\left|c_{i j}\right| \exp \left(a_{*} \cdot \vartheta\right)\right) .
$$

Then the w-periodic solution of the CNN models with the DEGPCD system (2.17a), with the respective constant coefficients, is globally exponentially stable.

Remark 3.32. When the the impulsive effects are absent, the stability result of Corollary 3.28 reduces to the stability result of [17, Theorem 3] with generalized piecewise alternately advanced and retarded argument and Corollary 3.30 reduces to the results of [15. Theorem 5.1] with generalized piecewise constant delay. We are able to see that the results obtained in this article extend and improve the results given in \begin{tabular}{|l|l|l|l|l|l|l|l|l|l|}
1 & 2 & 10 & 14 & 15 & 17
\end{tabular} . Remark 3.33. Recently, the existence and stability of the periodic solution to the ICNN models with piecewise constant argument have been studied extensively. However, we do not find related works concerning the periodic solution for impulsive cellular neural network models with piecewise alternately advanced and retarded argument of generalized type.

From this point, the model considered in this paper is more general than the existing the ICNN models and the CNN models with piecewise constant argument such as those in References $1,2,10,14,15,17$. 


\section{Illustrative examples with simulations}

In this section we should present two illustrative examples with simulations for our proposed results.

Example 4.1. Consider the following ICNN models with the IDEPCAG system

$$
\left\{\begin{aligned}
x_{1}^{\prime}= & -a_{1}(\cdot) x_{1}+b_{11}(\cdot) f_{1}\left(x_{1}\right)+b_{12}(\cdot) f_{2}\left(x_{2}\right) \\
& +c_{12}(\cdot) g_{2}\left(x_{2}(\gamma(\cdot))\right)+c_{13}(\cdot) g_{3}\left(x_{3}(\gamma(\cdot))+d_{1}(\cdot),\right. \\
x_{2}^{\prime}= & -a_{2}(\cdot) x_{2}+b_{21}(\cdot) f_{1}\left(x_{1}\right)+b_{23}(\cdot) f_{3}\left(x_{3}\right) \\
& +c_{21}(\cdot) g_{1}\left(x_{1}(\gamma(\cdot))\right)+c_{22}(\cdot) g_{2}\left(x_{2}(\gamma(\cdot))\right)+d_{2}(\cdot), \\
x_{3}^{\prime}= & -a_{3}(\cdot) x_{2}+b_{31}(\cdot) f_{1}\left(x_{1}\right)+b_{32}(\cdot) f_{2}\left(x_{2}\right) \\
& +c_{31}(\cdot) g_{1}\left(x_{1}(\gamma(\cdot))\right)+c_{33}(\cdot) g_{3}\left(x_{3}(\gamma(\cdot))\right)+d_{3}(\cdot), \\
& \left\{\begin{array}{l}
\Delta x_{1}\left(t_{\kappa}\right)=\mathfrak{J}_{1 \kappa}\left(x_{1}\left(t_{\kappa}^{-}\right)\right), \\
\Delta x_{2}\left(t_{\kappa}\right)=\mathfrak{J}_{2 \kappa}\left(x_{2}\left(t_{\kappa}^{-}\right)\right), \\
\Delta x_{3}\left(t_{\kappa}\right)=\mathfrak{J}_{3 \kappa}\left(x_{3}\left(t_{\kappa}^{-}\right)\right),
\end{array}\right.
\end{aligned}\right.
$$

where

$$
\begin{array}{cll}
a_{1}(t)=1.1+0.1 \cos \left(\frac{16}{3} t\right), & a_{2}(t)=0.7+0.1 \sin \left(\frac{16}{3} t\right), & a_{3}(t)=0.85-0.05 \sin \left(\frac{16}{3} t\right), \\
b_{11}(t)=0.15+0.1 \sin \left(\frac{16}{3} t\right), & b_{12}(t)=0.1+0.05 \cos \left(\frac{16}{3} t\right), & b_{21}(t)=0.1+0.05 \sin \left(\frac{16}{3} t\right), \\
b_{23}(t)=0.25+0.1 \cos \left(\frac{16}{3} t\right), & b_{31}(t)=0.25+0.1 \sin \left(\frac{16}{3} t\right), & b_{32}(t)=0.15+0.1 \cos \left(\frac{16}{3} t\right), \\
c_{12}(t)=0.1+0.05 \sin \left(\frac{16}{3} t\right), & c_{13}(t)=0.25+0.1 \cos \left(\frac{16}{3} t\right), & c_{21}(t)=0.15+0.1 \sin \left(\frac{16}{3} t\right), \\
c_{22}(t)=0.25+0.1 \cos \left(\frac{16}{3} t\right), & c_{31}(t)=0.1+0.05 \sin \left(\frac{16}{3} t\right), & c_{33}(t)=0.15+0.1 \cos \left(\frac{16}{3} t\right), \\
d_{1}(t)=0.8+0.1 \sin \left(\frac{16}{3} t\right), & d_{2}(t)=0.5+0.1 \cos \left(\frac{16}{3} t\right), & d_{3}(t)=0.6+0.1 \cos \left(\frac{16}{3} t\right),
\end{array}
$$

and $\gamma(t)=\frac{3 \pi}{8} \kappa-\frac{\pi}{4}$, if $\frac{3 \pi}{8}(\kappa-1) \leq t<\frac{3 \pi}{8} \kappa, \kappa \in \mathbb{N}$.

The output functions are

$$
\begin{aligned}
f_{1}\left(x_{1}(t)\right) & =-\cos \left(\frac{x_{1}(t)}{10}\right)+\frac{x_{1}(t)}{15}+1, & f_{2}\left(x_{2}(t)\right) & =\sin \left(\frac{x_{2}(t)}{6}\right)+\frac{x_{2}(t)}{12}, \\
f_{3}\left(x_{3}(t)\right) & =\cos \left(\frac{x_{3}(t)}{12}\right)+\frac{x_{3}(t)}{24}-1, & g_{1}\left(x_{1}(\gamma(t))\right) & =\sin \left(\frac{x_{1}(\gamma(t))}{4}\right), \\
g_{2}\left(x_{2}(\gamma(t))\right) & =-\cos \left(\frac{x_{2}(\gamma(t))}{8}\right)+1, & & g_{3}\left(x_{3}(\gamma(t))\right)=\sin \left(\frac{x_{3}(\gamma(t))}{3}\right) .
\end{aligned}
$$


The impulsive functions are

$$
\begin{aligned}
& \mathfrak{J}_{1 \kappa}\left(x_{1}\left(t_{\kappa}^{-}\right)\right)=\mathfrak{J}_{1 \kappa}\left(x_{1}\left(\frac{3 \pi}{8}(\kappa-1)^{-}\right)\right)=\frac{x_{1}\left(\frac{3 \pi}{8}(\kappa-1)^{-}\right)}{5}-0.5, \\
& \mathfrak{J}_{2 \kappa}\left(x_{2}\left(t_{\kappa}^{-}\right)\right)=\mathfrak{J}_{2 \kappa}\left(x_{2}\left(\frac{3 \pi}{8}(\kappa-1)^{-}\right)\right)=\frac{x_{2}\left(\frac{3 \pi}{8}(\kappa-1)^{-}\right)}{8}+0.3, \\
& \mathfrak{J}_{3 \kappa}\left(x_{3}\left(t_{\kappa}^{-}\right)\right)=\mathfrak{J}_{3 \kappa}\left(x_{3}\left(\frac{3 \pi}{8}(\kappa-1)^{-}\right)\right)=\frac{x_{3}\left(\frac{3 \pi}{8}(\kappa-1)^{-}\right)}{6}-0.4 .
\end{aligned}
$$

We can easily obtain that the maximal distance $\vartheta_{k}^{+}=\gamma_{k}-t_{k}=\frac{\pi}{8}, \vartheta_{k}^{-}=t_{k+1}-\gamma_{k}=\frac{\pi}{4}$, $\vartheta=\vartheta_{k}=\frac{3 \pi}{8}, \mathfrak{L}_{1}^{f}=\mathfrak{L}_{3 \kappa}^{J}=\frac{1}{6}, \mathfrak{L}_{2}^{f}=\mathfrak{L}_{1}^{g}=\frac{1}{4}, \mathfrak{L}_{3}^{f}=\mathfrak{L}_{2}^{g}=\mathfrak{L}_{2 \kappa}^{J}=\frac{1}{8}, \mathfrak{L}_{3}^{g}=\frac{1}{3}, \mathfrak{L}_{1 \kappa}^{J}=\frac{1}{5}, \mathfrak{L}_{\kappa}^{J}=\frac{1}{5}$, $\sup _{\kappa \in \mathbb{N}} \frac{\ln \left(1+\mathfrak{L}_{\kappa}^{J}\right)}{\vartheta_{\kappa}} \approx 0.15476, \bar{a}_{*}=0.8, \underline{a}_{*}=0.6$ and $\left\{t_{i}\right\}_{i \in \mathbb{N}},\left\{\gamma_{i}\right\}_{i \in \mathbb{N}},\left\{J_{k}\right\}_{k \in \mathbb{N}}$ satisfy the $\left(\frac{3 \pi}{8}, 1\right)$ condition. Moreover,

\begin{tabular}{|c|c|c|c|c|c|c|c|c|c|}
\hline & $a_{1}(\cdot)$ & $a_{2}(\cdot)$ & $a_{3}(\cdot)$ & $b_{11}(\cdot)$ & $b_{12}(\cdot)$ & $b_{21}(\cdot)$ & $b_{23}(\cdot)$ & $b_{31}(\cdot)$ & $b_{32}(\cdot)$ \\
\hline $\max _{t \in \mathbb{R}^{+}}$ & 1.2 & 0.8 & 0.9 & 0.25 & 0.15 & 0.15 & 0.35 & 0.35 & 0.25 \\
\hline $\min _{t \in \mathbb{R}^{+}}$ & 1.0 & 0.6 & 0.8 & 0.05 & 0.05 & 0.05 & 0.15 & 0.15 & 0.05 \\
\hline & $c_{12}(\cdot)$ & $c_{13}(\cdot)$ & $c_{21}(\cdot)$ & $c_{22}(\cdot)$ & $c_{31}(\cdot)$ & $c_{33}(\cdot)$ & $d_{1}(\cdot)$ & $d_{2}(\cdot)$ & $d_{3}(\cdot)$ \\
\hline $\max _{t \in \mathbb{R}^{+}}$ & 0.15 & 0.35 & 0.25 & 0.35 & 0.15 & 0.25 & 0.9 & 0.6 & 0.7 \\
\hline $\min _{t \in \mathbb{R}^{+}}$ & 0.05 & 0.15 & 0.05 & 0.15 & 0.05 & 0.05 & 0.7 & 0.4 & 0.5 \\
\hline
\end{tabular}

It follows that

(a)

$$
\begin{aligned}
& \kappa^{+}=\max _{1 \leq i \leq 3}\left\{\sup _{i(\tau) \leq \kappa}\left(\frac{1-e^{-\underline{a}_{i} \cdot \vartheta_{\kappa}^{+}}}{\underline{a}_{i}}\right)\left[\sum_{j=1}^{n} \mathfrak{L}_{j}^{f}\left|\bar{b}_{i j}\right|+\sum_{j=1}^{n} \mathfrak{L}_{j}^{g}\left|\bar{c}_{i j}\right|\right]\right\} \approx 0.28009<1, \\
& \kappa^{-}=\max _{1 \leq i \leq 3}\left\{\sup _{1 \leq \kappa \leq i(\tau)}\left(\frac{e^{\bar{a}_{i} \cdot \vartheta_{\kappa}^{-}}-1}{\bar{a}_{i}}\right)\left[\sum_{j=1}^{n} \mathfrak{L}_{j}^{f}\left|\bar{b}_{i j}\right|+\sum_{j=1}^{n} \mathfrak{L}_{j}^{g}\left|\bar{c}_{i j}\right|\right]\right\} \approx 0.08144<1 .
\end{aligned}
$$

(b)

$$
\begin{aligned}
& \frac{\exp \left(\bar{a}_{1} \omega\right)}{\exp \left(\underline{a}_{1} \omega\right)-1}\left[\left(\mathfrak{L}_{1}^{f}\left|\bar{b}_{11}\right|+\mathfrak{L}_{2}^{f}\left|\bar{b}_{12}\right|+\mathfrak{L}_{2}^{g}\left|\bar{c}_{12}\right|+\mathfrak{L}_{3}^{g}\left|\bar{c}_{13}\right|\right) \omega+\mathfrak{L}_{1}^{J}\right] \approx 0.92827<1, \\
& \frac{\exp \left(\bar{a}_{2} \omega\right)}{\exp \left(\underline{a}_{2} \omega\right)-1}\left[\left(\mathfrak{L}_{1}^{f}\left|\bar{b}_{21}\right|+\mathfrak{L}_{3}^{f}\left|\bar{b}_{23}\right|+\mathfrak{L}_{1}^{g}\left|\bar{c}_{21}\right|+\mathfrak{L}_{2}^{g}\left|\bar{c}_{22}\right|\right) \omega+\mathfrak{L}_{2}^{J}\right] \approx 0.89702<1, \\
& \frac{\exp \left(\bar{a}_{3} \omega\right)}{\exp \left(\underline{a}_{3} \omega\right)-1}\left[\left(\mathfrak{L}_{1}^{f}\left|\bar{b}_{31}\right|+\mathfrak{L}_{2}^{f}\left|\bar{b}_{32}\right|+\mathfrak{L}_{1}^{g}\left|\bar{c}_{31}\right|+\mathfrak{L}_{3}^{g}\left|\bar{c}_{33}\right|\right) \omega+\mathfrak{L}_{3}^{J}\right] \approx 0.89348<1 .
\end{aligned}
$$


(c)

$$
\begin{aligned}
& \mu_{1}=\sum_{j=1}^{3} \mathfrak{L}_{j}^{f}\left|b_{1 j}\right|+\sum_{j=1}^{3} \mathfrak{L}_{j}^{g}\left|c_{1 j}\right| \frac{e^{\bar{a}_{*} \cdot \vartheta^{-}}}{1-\widehat{\nu}} \approx 0.43111<0.44524 \approx \underline{a}_{*}-\sup _{\kappa \in \mathbb{N}} \frac{\ln \left(1+\mathfrak{L}_{\kappa}^{J}\right)}{\vartheta_{\kappa}}, \\
& \mu_{2}=\sum_{j=1}^{3} \mathfrak{L}_{j}^{f}\left|b_{2 j}\right|+\sum_{j=1}^{3} \mathfrak{L}_{j}^{g}\left|c_{2 j}\right| \frac{e^{\bar{a}_{*} \cdot \vartheta^{-}}}{1-\widehat{\nu}} \approx 0.27316<0.44524 \approx \underline{a}_{*}-\sup _{\kappa \in \mathbb{N}} \frac{\ln \left(1+\mathfrak{L}_{\kappa}^{J}\right)}{\vartheta_{\kappa}}
\end{aligned}
$$

and

$$
\mu_{3}=\sum_{j=1}^{3} \mathfrak{L}_{j}^{f}\left|b_{3 j}\right|+\sum_{j=1}^{3} \mathfrak{L}_{j}^{g}\left|c_{3 j}\right| \frac{e^{\bar{a}_{*} \cdot \vartheta^{-}}}{1-\widehat{\nu}} \approx 0.36267<0.44524 \approx \underline{a}_{*}-\sup _{\kappa \in \mathbb{N}} \frac{\ln \left(1+\mathfrak{L}_{\kappa}^{J}\right)}{\vartheta_{\kappa}},
$$

where

$$
\widehat{\nu}=\sup _{k \in \mathbb{N}}\left[\max _{1 \leq i \leq 3}\left(\sum_{j=1}^{3} \mathfrak{L}_{j}^{f}\left|\bar{b}_{i j}\right|+\sum_{j=1}^{3} \mathfrak{L}_{j}^{g}\left|\bar{c}_{i j}\right|\left(\frac{1-e^{-\underline{a}_{*} \cdot \vartheta_{k}^{+}}}{\underline{a}_{*}}\right)\right) \cdot \vartheta_{k}^{+}\right] \approx 0.06344<1 .
$$

Then we have

$$
\underline{a}_{*}-\max _{1 \leq i \leq 3} \bar{\mu}_{i}-\sup _{\kappa \in \mathbb{N}} \frac{\ln \left(1+\mathfrak{L}_{\kappa}^{J}\right)}{\vartheta_{\kappa}} \approx 0.08256>0 .
$$

One can see that all conditions $\left.\left(\mathrm{L}_{1}\right),\left(\mathrm{L}_{2}\right),(\mathrm{P}), 22.15\right),(3.6),(3.13)$ and $(3.14)$ in Corollary 3.22 are satisfied. Therefore, the ICNN models with the IDEPCAG system 4.1a)4.1b has a unique globally exponentially stable $\frac{3 \pi}{8}$-periodic solution. The simulation of the unique $\frac{3 \pi}{8}$-periodic solution of the ICNN models $4.1 \mathrm{a}-4.1 \mathrm{~b}$ with and without impulses, are shown in Figures 4.14 .8 .

For the simulation, the initial states $\left(x_{1}(0), x_{2}(0), x_{3}(0)\right)^{T}$ are given by the random function. Figures 4.14 .6 show that the conditions obtained in this article are valid for the ICNN models with the IDEPCAG system 4.1a) $4.1 \mathrm{~b}$.

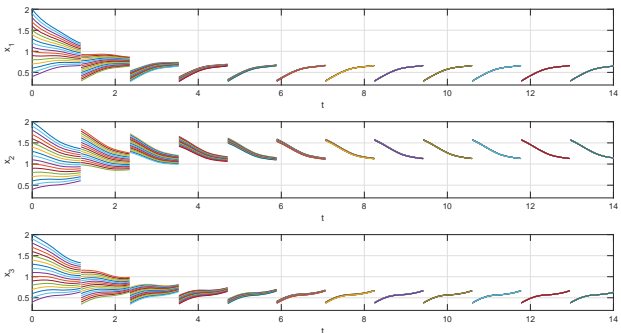

Figure 4.1: Some trajectories uniformly convergent to the unique exponentially stable $\frac{3 \pi}{8}$-periodic solution of the ICNN models with the IDEPCAG system 4.1a) $-4.1 \mathrm{~b}$.

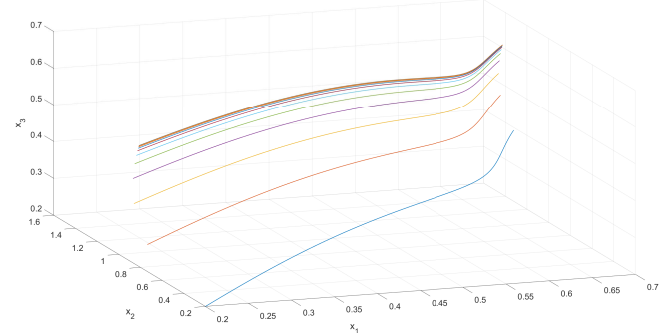

Figure 4.2: Phase plots of state variable $\left(x_{1}, x_{2}, x_{3}\right)$ in the ICNN models with the IDEPCAG system 4.1a-4.1b with the initial condition $(0.2,0.2,0.2)^{T}$. 


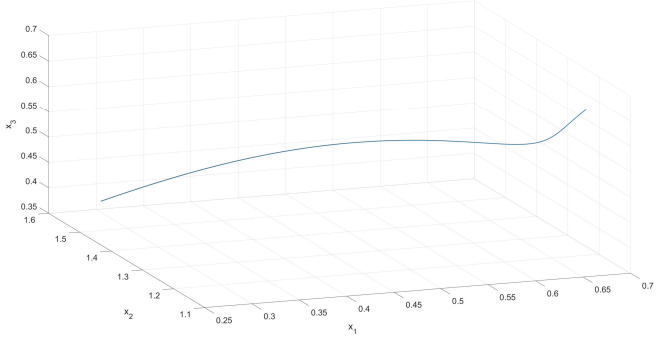

Figure 4.3: Phase plots of state variable $\left(x_{1}, x_{2}, x_{3}\right)$ in the ICNN models with the IDEPCAG system 4.1a(4.1b) with the initial condition $(0.29529,1.5702,0.37765)^{T}$.

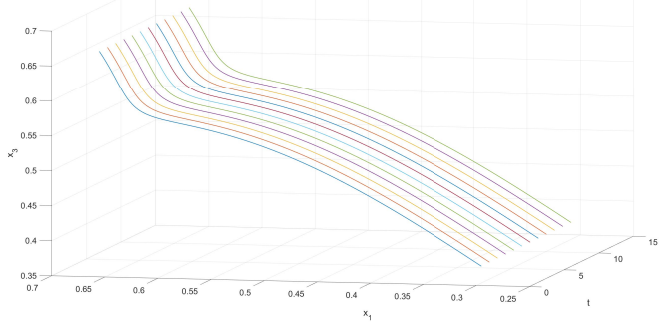

Figure 4.5: Phase plots of state variable $\left(t, x_{1}, x_{3}\right)$ in the ICNN models with the IDEPCAG system 4.1a-4.1b).
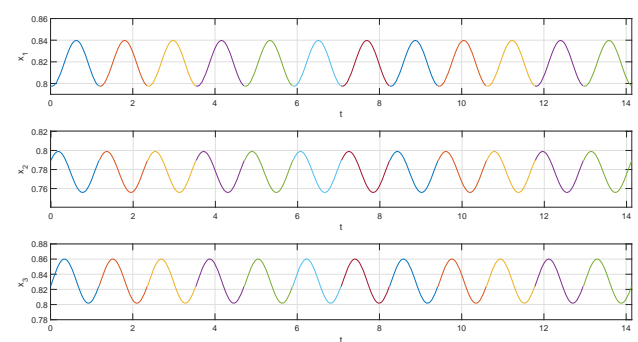

Figure 4.7: $\frac{3 \pi}{8}$-periodic solution of the CNN models with the DEPCAG system 4.1a) for $t \in[0,14]$ with the initial value $(0.7979,0.7895,0.8238)^{T}$.

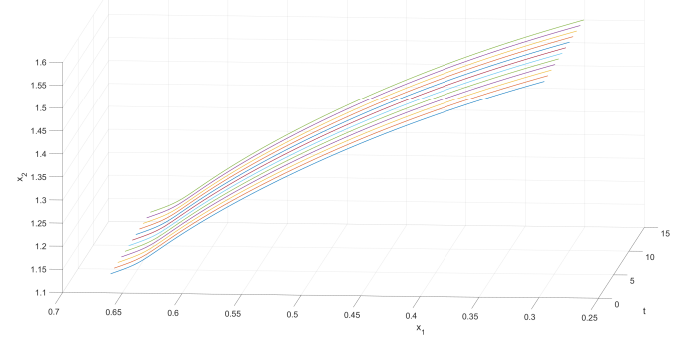

Figure 4.4: Phase plots of state variable $\left(t, x_{1}, x_{2}\right)$ in the ICNN models with the IDEPCAG system 4.1a-4.1b.

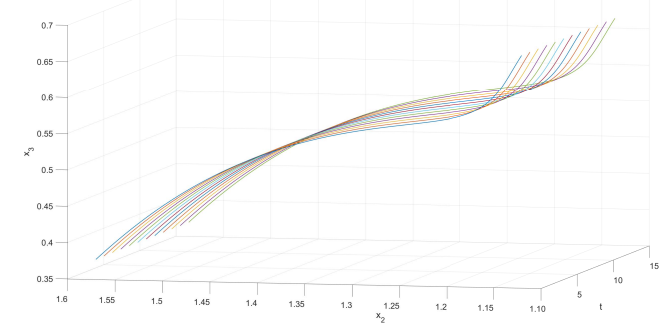

Figure 4.6: Phase plots of state variable $\left(t, x_{2}, x_{3}\right)$ in the ICNN models with the IDEPCAG system 4.1a)-4.1b).

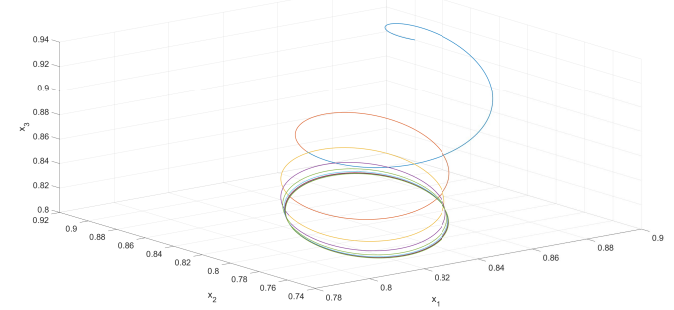

Figure 4.8: Trajectories uniformly convergent to the unique exponentially stable $\frac{3 \pi}{8}$-periodic solution of the $\mathrm{CNN}$ models with the DEPCAG system 4.1a with the initial value $(0.9,0.9,0.9)^{T}$. 
Numerical simulations confirm that the proposed conditions in our results are effective for the ICNN models with the IDEPCAG system (4.1a)-(4.1b).

Example 4.2. Let $a_{1}(t) \equiv 1.2, a_{2}(t) \equiv 0.7, b_{11}(t)=c_{12}(t) \equiv 0.14, b_{12}(t) \equiv 0.11, b_{21}(t) \equiv$ $0.125, b_{22}(t)=c_{22}(t) \equiv 0.12, c_{11}(t) \equiv 0.15, c_{21}(t) \equiv 0.13$, and $d_{1}(t) \equiv 3, d_{2}(t) \equiv 2$. Then the ICNN models with the IDEPCAG system 2.1a $-2.1 \mathrm{~b}$ reduces to the following system

$$
\begin{aligned}
\frac{d x(t)}{d t}= & -\left(\begin{array}{cc}
1.2 & 0 \\
0 & 0.7
\end{array}\right)\left(\begin{array}{l}
x_{1}(t) \\
x_{2}(t)
\end{array}\right)+\left(\begin{array}{cc}
0.14 & 0.11 \\
0.125 & 0.12
\end{array}\right)\left(\begin{array}{c}
\tanh \left(\frac{x_{1}(t)}{8}\right) \\
\tanh \left(\frac{x_{2}(t)}{5}\right)
\end{array}\right) \\
& +\left(\begin{array}{ll}
0.15 & 0.14 \\
0.13 & 0.12
\end{array}\right)\left(\frac{\left|x_{1}\left(4\left[\frac{t+3}{4}\right]\right)+1\right|-\left|x_{1}\left(4\left[\frac{t+3}{4}\right]\right)-1\right|}{14}\right)+\left(\begin{array}{l}
3 \\
2
\end{array}\right), \\
\left.\Delta x\right|_{t=k}= & \left(\begin{array}{l}
J_{1 k}\left(x_{1}\left(k^{-}\right)\right) \\
J_{2 k}\left(x_{2}\left(k^{-}\right)\right)
\end{array}\right)=\left(\begin{array}{l}
(-1)^{k} \frac{x_{1}\left(k^{-}\right)}{6}+2.5 \\
(-1)^{k} \frac{x_{2}\left(k^{-}\right)}{5}+3.5
\end{array}\right), \quad k \in \mathbb{N} .
\end{aligned}
$$

We can easily obtain that $t_{1}=\gamma_{1}=0, t_{i}=4(i-2)+1, \gamma_{i}=4(i-1), i \geq 2$, $\vartheta^{+}=\vartheta_{k}^{+}=\gamma_{k}-t_{k}=3, \vartheta^{-}=\vartheta_{k}^{-}=t_{k+1}-\gamma_{k}=1, \vartheta=\vartheta_{k}=4, k \in \mathbb{N}, \mathfrak{L}_{1}^{f}=\frac{1}{8}, \mathfrak{L}_{2}^{f}=\frac{1}{5}$, $\mathfrak{L}_{1}^{g}=\frac{1}{7}, \mathfrak{L}_{2}^{g}=\frac{1}{8}, \mathfrak{L}_{1 \kappa}^{J}=\frac{1}{6}, \mathfrak{L}_{2 \kappa}^{J}=\frac{1}{5}, \mathfrak{L}_{\kappa}^{J}=\frac{1}{5}, \sup _{\kappa \in \mathbb{N}} \frac{\ln \left(1+\mathfrak{L}_{\kappa}^{J}\right)}{\vartheta_{\kappa}} \approx 0.04558, a_{*}=0.7$ and $\left\{t_{i}\right\}_{i \in \mathbb{N}},\left\{\gamma_{i}\right\}_{i \in \mathbb{N}},\left\{J_{k}\right\}_{k \in \mathbb{N}}$ satisfy the $(8,2)$ condition.

It follows that

(a)

$$
\kappa^{+}=\max _{1 \leq i \leq 2}\left\{\sup _{i(\tau) \leq \kappa}\left(\frac{1-e^{-a_{i} \cdot \vartheta_{\kappa}^{+}}}{a_{i}}\right)\left[\sum_{j=1}^{2} \mathfrak{L}_{j}^{f}\left|b_{i j}\right|+\sum_{j=1}^{2} \mathfrak{L}_{j}^{g}\left|c_{i j}\right|\right]\right\} 0.151636<1
$$

and

$$
\kappa^{-}=\max _{1 \leq i \leq 2}\left\{\sup _{1 \leq \kappa \leq i(\tau)}\left(\frac{e^{a_{i} \cdot \vartheta_{\kappa}^{-}}-1}{a_{i}}\right)\left[\sum_{j=1}^{2} \mathfrak{L}_{j}^{f}\left|b_{i j}\right|+\sum_{j=1}^{2} \mathfrak{L}_{j}^{g}\left|c_{i j}\right|\right]\right\} 0.091761<1 .
$$

(b)

$$
\frac{\exp \left(a_{1} \omega\right)}{\exp \left(a_{1} \omega\right)-1}\left[\sum_{j=1}^{2}\left(\mathcal{L}_{j}^{f}\left|b_{1 j}\right|+\mathcal{L}_{j}^{g}\left|c_{1 j}\right|\right) \omega+2 \cdot \mathcal{L}_{1}^{J}\right] \approx 0.96082<1
$$

and

$$
\frac{\exp \left(a_{2} \omega\right)}{\exp \left(a_{2} \omega\right)-1}\left[\sum_{j=1}^{2}\left(\mathcal{L}_{j}^{f}\left|b_{2 j}\right|+\mathcal{L}_{j}^{g}\left|c_{2 j}\right|\right) \omega+2 \cdot \mathcal{L}_{2}^{J}\right] \approx 0.98922<1
$$


(c)

$$
\mu_{1}=\sum_{j=1}^{2} \mathfrak{L}_{j}^{f}\left|b_{1 j}\right|+\sum_{j=1}^{2} \mathfrak{L}_{j}^{g}\left|c_{1 j}\right| \frac{e^{a_{*} \cdot \vartheta^{-}}}{1-\widehat{\nu}} \approx 0.143349<0.654419 \approx a_{*}-\sup _{\kappa \in \mathbb{N}} \frac{\ln \left(1+\mathfrak{L}_{\kappa}^{J}\right)}{\vartheta_{\kappa}}
$$

and

$$
\mu_{2}=\sum_{j=1}^{3} \mathfrak{L}_{j}^{f}\left|b_{2 j}\right|+\sum_{j=1}^{3} \mathfrak{L}_{j}^{g}\left|c_{2 j}\right| \frac{e^{a_{*} \cdot \vartheta^{-}}}{1-\widehat{\nu}} \approx 0.129183<0.654419 \approx a_{*}-\sup _{\kappa \in \mathbb{N}} \frac{\ln \left(1+\mathfrak{L}_{\kappa}^{J}\right)}{\vartheta_{\kappa}},
$$

where

$$
\widehat{\nu}=\sup _{k \in \mathbb{N}}\left[\max _{1 \leq i \leq 2}\left(\sum_{j=1}^{2} \mathfrak{L}_{j}^{f}\left|b_{i j}\right|+\sum_{j=1}^{2} \mathfrak{L}_{j}^{g}\left|c_{i j}\right|\left(\frac{1-e^{-a_{*} \cdot \vartheta_{k}^{+}}}{a_{*}}\right)\right) \cdot \vartheta_{k}^{+}\right] \approx 0.245133<1
$$

Then

$$
a_{*}-\max _{1 \leq i \leq 2} \mu_{i}-\sup _{\kappa \in \mathbb{N}} \frac{\ln \left(1+\mathfrak{L}_{\kappa}^{J}\right)}{\vartheta_{\kappa}} \approx 0.51107>0 .
$$

In this case, we can easily verify that all conditions $\left(\mathrm{L}_{1}\right),\left(\mathrm{L}_{2}\right),(\mathrm{P}),(2.16),(3.7), 3.15$ and 3.16 ) of Corollary 3.23 are satisfied. Thus, according to Corollary 3.23, the ICNN models with the IDEPCAG system with constant coefficients $4.2 \mathrm{a}-(4.2 \mathrm{~b}$ ) has a unique 8-periodic solution and all other solution of system converge exponentially to it as $t \rightarrow \infty$.

The numerical simulations, showing the convergence of the 8-periodic solution of the ICNN models with the IDEPCAG system with constant coefficients 4.2a $-4.2 \mathrm{~b}$, are given in Figures 4.94 .12 .
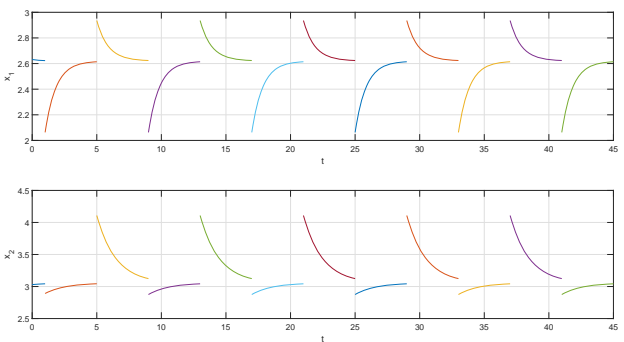

Figure 4.9: 8-periodic solution of the ICNN models with the IDEPCAG system 4.2a - 4.2b with the initial condition $(2.6315,3.0302)^{T}$.

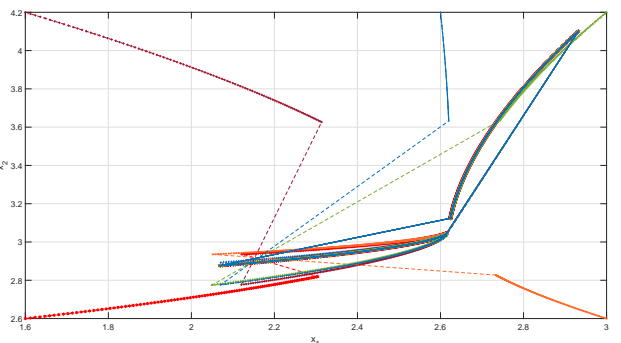

Figure 4.10: Exponential convergence of five trajectories towards an 8-periodic solution of the ICNN models with the IDEPCAG system 4.2a)-4.2b). 


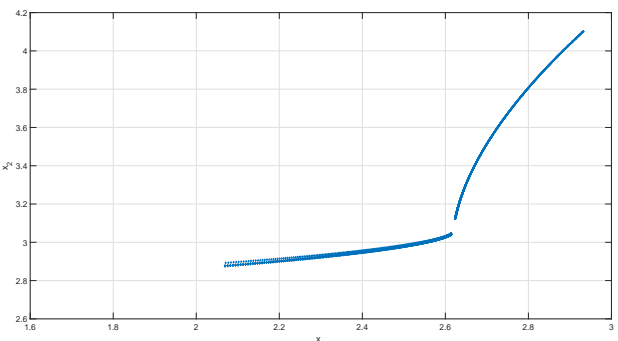

Figure 4.11: Phase plane behavior of the state variables $x_{1}$ and $x_{2}$ for the ICNN models with the IDEPCAG system 4.2a - 4.2b with the initial condition $(2.6315,3.0302)^{T}$.

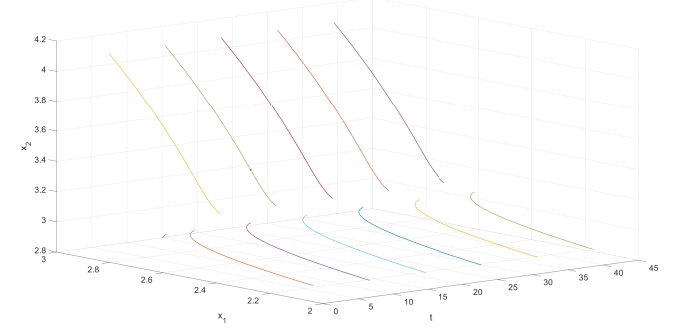

Figure 4.12: Phase plots of state variable $\left(t, x_{1}, x_{2}\right)$ in the ICNN models with the IDEPCAG system 4.2a-4.2b).

Remark 4.3. When considering system 4.2a $-4.2 \mathrm{~b}$ with generalized piecewise constant delay, the parameters of the system $4.2 \mathrm{a}-(4.2 \mathrm{~b}$ ) do not satisfy Theorem 4.2 in [1]. This implies that the results in the present paper are less conservative than the results in [1].

\section{Conclusions}

In this paper, the unique globally exponentially stable periodic solution for the impulsive cellular neural network models with piecewise alternately advanced and retarded argument of generalized type have been investigated. By using the equivalent integral equation, a new IDEPCAG's Gronwall inequality and Banach fixed-point theorem, some new sufficient conditions have been developed to ensure the existence, uniqueness and global exponential stability of the periodic solution for general non-autonomous ICNN models with the IDEPCAG system. The proposed criteria for the existence and stability theorems are easily tested by analyzing multiple relationships between neural network parameters and Lipschitz constants without asking for the conditions of differentiability, monotonicity or boundedness. Based on the proposed approach, it is unnecessary to utilize Razumikhin-type technique or construct a Lyapunov function that is applied from the previous literature. Moreover, illustrative simulation examples show that the approach used is more efficient and extend the results of the previous literature $[1,10,14,17]$.

\section{Acknowledgments}

The author thanks the referees very much for their valuable suggestions which made this paper much improved. 


\section{References}

[1] M. U. Akhmet and E. Yımaz, Impulsive Hopfield-type neural network system with piecewise constant argument, Nonlinear Anal. Real World Appl. 11 (2010), no. 4, $2584-2593$.

[2] _ Neural Networks with Discontinuous/Impact Activations, Nonlinear Systems and Complexity 9, Springer, New York, 2014.

[3] E. Barone and C. Tebaldi, Stability of equilibria in a neural network model, Math. Methods Appl. Sci. 23 (2000), no. 13, 1179-1193.

[4] S. Busenberg and K. Cooke, Vertically Transmitted Diseases: Models and dynamics, Biomathematics 23, Springer-Verlag, Berlin, 1993.

[5] J.-D. Cao, Global asymptotic stability of neural networks with transmission delays, Internat. J. Systems Sci. 31 (2000), no. 10, 1313-1316.

[6] S. Castillo, M. Pinto and R. Torres, Asymptotic formulae for solutions to impulsive differential equations with piecewise constant argument of generalized type, Electron. J. Differential Equations 2019 (2019), Paper No. 40, 22 pp.

[7] T. Chen, Global exponential stability of delayed Hopfield neural networks, Neural Networks 14 (2001), no. 8, 977-980.

[8] K.-S. Chiu, Existence and global exponential stability of equilibrium for impulsive cellular neural network models with piecewise alternately advanced and retarded argument, Abstr. Appl. Anal. 2013 (2013), Art. ID 196139, 13 pp.

[9] _ On generalized impulsive piecewise constant delay differential equations, Sci. China Math. 58 (2015), no. 9, 1981-2002.

[10] _ Exponential stability and periodic solutions of impulsive neural network models with piecewise constant argument, Acta Appl. Math. 151 (2017), 199-226.

[11] _ Asymptotic equivalence of alternately advanced and delayed differential systems with piecewise constant generalized arguments, Acta Math. Sci. Ser. B (Engl. Ed.) 38 (2018), no. 1, 220-236.

[12] _ Existence and global exponential stability of equilibrium for impulsive neural network models with generalized piecewise constant delay, to appear in Asian-Eur. J. Math. 2021. https://doi.org/10.1142/s1793557122500012 
[13] _ Green's function for impulsive periodic solutions in alternately advanced and delayed differential systems and applications, Commun. Fac. Sci. Univ. Ank. Ser. A1. Math. Stat. 70 (2021), no. 1, 15-37.

[14]__ Periodicity and stability analysis of impulsive neural network models with generalized piecewise constant delays, to appear in Discrete Contin. Dyn. Syst. Ser. B, 2021. https://doi.org/10.3934/dcdsb.2021060

[15] K.-S. Chiu and F. Córdova-Lepe, Global exponential periodicity and stability of neural network models with generalized piecewise constant delay, Math. Slovaca 71 (2021), no. $2,491-512$.

[16] K.-S. Chiu and T. Li, Oscillatory and periodic solutions of differential equations with piecewise constant generalized mixed arguments, Math. Nachr. 292 (2019), no. 10, 2153-2164.

[17] K.-S. Chiu, M. Pinto and J.-C. Jeng, Existence and global convergence of periodic solutions in recurrent neural network models with a general piecewise alternately advanced and retarded argument, Acta Appl. Math. 133 (2014), 133-152.

[18] L. O. Chua and L. Yang, Cellular neural networks: Theory, IEEE Trans. Circuits and Systems 35 (1988), no. 10, 1257-1272.

[19] K. Gopalsamy, Stability of artificial neural networks with impulses, Appl. Math. Comput. 154 (2004), no. 3, 783-813.

[20] Z. Huang, X. Wang and F. Gao, The existence and global attractivity of almost periodic sequence solution of discrete-time neural networks, Phys. Lett. A 350 (2006), no. 3-4, $182-191$.

[21] Z. Huang, X. Wang and Y. Xia, A topological approach to the existence of solutions for nonlinear differential equations with piecewise constant argument, Chaos Solitons Fractals 39 (2009), no. 3, 1121-1131.

$[22] \_$Exponential attractor of $\kappa$-almost periodic sequence solution of discrete-time bidirectional neural networks, Simul. Model. Pract. Theory 18 (2010), no. 3, 317-337.

[23] Z. Huang, Y. Xia and X. Wang, The existence and exponential attractivity of $\kappa$-almost periodic sequence solution of discrete time neural networks, Nonlinear Dynam. 50 (2007), no. 1-2, 13-26.

[24] F. Karakoç, Asymptotic behaviour of a population model with piecewise constant argument, Appl. Math. Lett. 70 (2017), 7-13. 
[25] O. M. Kwona, S. M. Lee, J. H. Park and E. J. Cha, New approaches on stability criteria for neural networks with interval time-varying delays, Appl. Math. Comput. 218 (2012), no. 19, 9953-9964.

[26] T. Li, X. Yao, L. Wu and J. Li, Improved delay-dependent stability results of recurrent neural networks, Appl. Math. Comput. 218 (2012), no. 19, 9983-9991.

[27] Z. Liu and L. Liao, Existence and global exponential stability of periodic solution of cellular neural networks with time-varying delays, J. Math. Anal. Appl. 290 (2004), no. 1, 247-262.

[28] X.-Y. Lou and B.-T. Cui, Novel global stability criteria for high-order Hopfield-type neural networks with time-varying delays, J. Math. Anal. Appl. 330 (2007), no. 1, $144-158$.

[29] S. Mohamad and K. Gopalsamy, Exponential stability of continuous-time and discretetime cellular neural networks with delays, Appl. Math. Comput. 135 (2003), no. 1, $17-38$.

[30] J. J. Nieto and R. Rodríguez-López, Second-order linear differential equations with piecewise constant arguments subject to nonlocal boundary conditions, Appl. Math. Comput. 218 (2012), no. 19, 9647-9656.

[31] J. H. Park, Global exponential stability of cellular neural networks with variable delays, Appl. Math. Comput. 183 (2006), no. 2, 1214-1219.

[32] M. Pinto, Asymptotic equivalence of nonlinear and quasi linear differential equations with piecewise constant arguments, Math. Comput. Modelling 49 (2009), no. 9-10, 1750-1758.

[33] M. Pinto and G. Robledo, Controllability and observability for a linear time varying system with piecewise constant delay, Acta Appl. Math. 136 (2015), 193-216.

[34] S. M. Shah and J. Wiener, Advanced differential equations with piecewise constant argument deviations, Internat. J. Math. Math. Sci. 6 (1983), no. 4, 671-703.

[35] B. Wang, S. Zhong and X. Liu, Asymptotical stability criterion on neural networks with multiple time-varying delays, Appl. Math. Comput. 195 (2008), no. 2, 809-818.

[36] J. Wiener, Differential equations with piecewise constant delays, in: Trends in Theory and Practice of Nonlinear Differential Equations (Arlington, Tex., 1982), 547-552, Lecture Notes in Pure and Appl. Math. 90, Dekker, New York, 1984. 
$[37]$ Generalized Solutions of Functional-differential Equations, World Scientific, Singapore, 1993.

[38] J. Wiener and V. Lakshmikantham, Differential equations with piecewise constant argument and impulsive equations, Nonlinear Stud. 7 (2000), no. 1, 60-69.

[39] Y. Xia, Z. Huang and M. Han, Existence of almost periodic solutions for forced perturbed systems with piecewise constant argument, J. Math. Anal. Appl. 333 (2007), no. $2,798-816$.

[40] B. Xu, X. Liu and X. Liao, Global exponential stability of high order Hopfield type neural networks, Appl. Math. Comput. 174 (2006), no. 1, 98-116.

[41] Y. Zhang, D. Yue and E. Tian, New stability criteria of neural networks with interval time-varying delay: A piecewise delay method, Appl. Math. Comput. 208 (2009), no. 1, 249-259.

[42] L. Zhou and G. Hu, Global exponential periodicity and stability of cellular neural networks with variable and distributed delays, Appl. Math. Comput. 195 (2008), no. 2, $402-411$.

\section{Kuo-Shou Chiu}

Departamento de Matemática, Facultad de Ciencias Básicas, Universidad Metropolitana de Ciencias de la Educación, José Pedro Alessandri 774, Santiago, Chile E-mail address: kschiu@umce.cl 